\title{
A myosin-Va tail fragment sequesters dynein light chains leading to apoptosis in melanoma cells
}

\author{
TC Izidoro-Toledo ${ }^{1,8}$, AC Borges ${ }^{1,8}$, DD Araújo ${ }^{1,2,3}$, DPS Leitão Mazzi ${ }^{1}$, FO Nascimento Júnior ${ }^{1}$, JF Sousa ${ }^{1,4}$, CP Alves ${ }^{1}$, APB Paiva ${ }^{1}$, \\ DM Trindade ${ }^{1,5}$, EV Patussi $^{1,6}$, PM Peixoto $^{7}, \mathrm{KW}^{1}$ Kinnally $^{7}$ and EM Espreafico ${ }^{*, 1}$
}

Previous studies proposed that myosin-Va regulates apoptosis by sequestering pro-apoptotic Bmf to the actin cytoskeleton through dynein light chain-2 (DLC2). Adhesion loss or other cytoskeletal perturbations would unleash Bmf, allowing it to bind and inhibit pro-survival Bcl2 proteins. Here, we demonstrated that overexpression of a myosin-Va medial tail fragment (MVaf) harboring the binding site for DLC2 dramatically decreased melanoma cell viability. Morphological and molecular changes, including surface blebbing, mitochondrial outer membrane permeabilization, cytochrome-c and Smac release, as well as caspase-9/-3 activation and DNA fragmentation indicated that melanoma cells died of apoptosis. Immobilized MVaf interacted directly with DLCs, but complexed MVaf/DLCs did not interact with Bmf. Overexpression of DLC2 attenuated MVaf-induced apoptosis. Thus, we suggest that, MVaf induces apoptosis by sequestering DLC2 and DLC1, thereby unleashing the pair of sensitizer and activator BH3-only proteins Bmf and Bim. Murine embryonic fibroblasts (MEFs) lacking Bim and Bmf or Bax and Bak were less sensitive to apoptosis caused by MVaf expression than wild-type MEFs, strengthening the putative role of the intrinsic apoptotic pathway in this response. Finally, MVaf expression attenuated B16-F10 solid tumor growth in mice, suggesting that this peptide may be useful as an apoptosis-inducing tool for basic and translational studies.

Cell Death and Disease (2013) 4, e547; doi:10.1038/cddis.2013.45; published online 21 March 2013

Subject Category: Cancer

Apoptosis is an active process of cell death essential for embryogenesis, tissue homeostasis, and elimination of damaged cells in multicellular organisms. Abnormalities in this process can initiate or contribute to the development of several diseases, such as autoimmune diseases, neurodegenerative disorders and cancer. ${ }^{1}$ The Bcl2 family of proteins constitutes a critical checkpoint in the apoptotic cascade. Cancer cells frequently express altered ratios of anti- to proapoptotic Bcl2 family members, which promote survival and confer resistance to therapy. ${ }^{2}$ The vertebrate dynein light chains (DLC) 1 and 2 (encoded by DYNLL1 and DYNLL2 genes) are $\sim 10 \mathrm{kDa}$ homodimeric hub proteins that interact with a large number of proteins involved in diverse biological functions, including the Bcl2 pro-apoptotic proteins Bim and $\mathrm{Bmf}$, as well as their respective molecular motor partners dynein and myosin-Va. ${ }^{3,4,5}$ Myosin- $\mathrm{Va}$ is an actin-based molecular motor, member of the class $\mathrm{V}$ myosins, which are comprised of highly related multi-domain proteins, encoded by three paralogous genes (MYO5A, MYO5B and MYO5C) in vertebrates. ${ }^{6}$ Myosin-Va is ubiquitously involved in the transport or tethering of a diverse array of cellular components to the actin cytoskeleton. ${ }^{7,8,9}$ Interestingly, myosin-Va has been suggested as a Bmf scavenger that releases the proapoptotic protein upon actin depolymerization or disruption of cell adhesion, ${ }^{10,12}$ in analogy with the mechanism proposed for the regulation of Bim by dynein and DLC1 in a manner dependent on microtubule integrity. ${ }^{11}$ Apoptosis triggered by loss of cell attachment constitutes a physiological form of cell death known as anoikis, which has a central role in controlling tissue homeostasis, and is believed to function as a major barrier against cancer. ${ }^{12,13,14}$ Therefore, the findings by Puthalakath et al. ${ }^{10}$ shed some light into how injuries that disrupt the actin cytoskeleton trigger cell death, and open many new questions, such as how these interactions are regulated, what are their roles in different cellular contexts, and how do malignant cells display resistance to anoikis. In the present work, we disrupted myosin-V/DLC2 interactions by overexpressing fragments derived from the medial tail region of myosin-Va. Surprisingly, some of the fragments worked as potent inducers of apoptosis in melanoma cells. These data provide further evidence of the role of myosin- $\mathrm{Va}$ and DLCs as critical pro-survival molecules.

\footnotetext{
${ }^{1}$ Department of Cellular and Molecular Biology and Pathogenic Bioagents, Faculty of Medicine of Ribeirão Preto, University of São Paulo, Ribeirão Preto, SP, Brazil; ${ }^{2}$ Faculty of Pharmaceutical Sciences of Ribeirão Preto, University of São Paulo, Ribeirão Preto, Brazil; ${ }^{3}$ University of Educational Foundation of Guaxupé - UNIFEG, Minas Gerais, Brazil; ${ }^{4}$ Vanderbilt University School of Medicine, Nashville, TN, USA; ${ }^{5}$ Brazilian Biosciences National Laboratory (LNBio) at Brazilian Center for Research in Energy and Materials (CNPEM), Campinas, Brazil; ${ }^{6}$ Department of Clinical Analysis and Biomedicine, State University of Maringá, Paraná, Brazil and ${ }^{7}$ New York University College of Dentistry, New York, NY, USA

*Corresponding author: EM Espreafico, Departamento de Biologia Celular e Molecular e Bioagentes Patogênicos, Faculdade de Medicina de Ribeirão Preto, USP, Av. Bandeirantes, 3900, Ribeirão Preto 14049-900, SP, Brazil. Tel: +55 163602 3348; Fax: + 55163633 1786; E-mail: emesprea@fmrp.usp.br

${ }^{8}$ These authors contributed equally to this work.

Keywords: Myosin-Va; DLC1/DLC2; apoptosis; melanoma; Bmf

Abbreviations: DLC1, Dynein light chain-1 (DYNLL1); DLC2, Dynein light chain-2 (DYNLL2); MT, medial tail; MVaf, myosin-Va medial tail fragment; MVcf, myosin-Vc fragment; EGF, epidermal growth factor; MOMP, mitochondrial outer membrane permeabilization; EGFP, enhanced green fluorescent protein; GST, glutathione-S-transferase; MBP, maltose-binding protein; MEFs, murine embryonic fibroblasts; FBS, fetal bovine serum; WT, wild-type

Received 11.10.12; revised 26.1.13; accepted 28.1.13; Edited by A Stephanou
} 


\section{Results}

Myosin-Va medial tail fragment impairs melanoma cell viability in an exon-B-dependent manner. To determine the impact of DLC2-myosin-Va interaction in melanoma cell viability, the effects of expressing myosin-Va fragments (MVaf), which include the DLC2-binding domain, ${ }^{5,15}$ were determined. Of three fragments generated, two contain exon B (MVaf1 and MVaf2) and one does not (MVaf3). A myosinVc fragment (MVcf) of a region nearby, which does not include exon B, was also generated (Figure 1a, and Supplementary Figure S1-A). The effects of MVaf1 and MVcf expression on proliferation rates were evaluated (Figures $1 \mathrm{~b}-\mathrm{c}$ ). The number of cells expressing enhanced green fluorescent protein (EGFP)-MVaf1 decayed drastically after $18 \mathrm{~h}$ of transfection, in contrast to EGFP-MVcf or EGFP. Even so, cultures expressing EGFP-MVcf and EGFP experienced a growth delay, which may have been caused by confluence or an underestimation of cell number in the more populated (control) plates under the stereomicroscope. In addition, attenuation of growth is less evident for EGFP than EGFP-MVcf-expressing cells, although cells undergoing apoptotic death were not observed (Supplementary Figure S1-E). Concomitant to cell loss, EGFP-MVaf1 protein levels progressively declined, whereas EGFP increased (Figure 1d). Propidium iodide (PI) labeled a high percentage of cells in the EGFP-MVaf1-transfected cultures (Figure 1e), indicating that loss of fluorescent signal was due to cell death. To investigate whether cell death was triggered by a specific sequence within MVaf1, we analyzed the effect of two additional constructs, EGFP-MVaf2 and EGFP-MVaf3. Interestingly, EGFP-MVaf2 expression caused a large drop in cell number, whereas EGFP-MVaf3 expression showed negligible effects (Figures $1 \mathrm{f}$ and $\mathrm{g}$ ). In contrast to MVaf3, MVaf1 and MVaf2 contain exon B, indicating that the presence of exon B was required for cell death. Conversely, exon $D$, which is partly present in MVaf1 but not in MVaf2 and MVaf3, was not required.

MVaf1 expression induces apoptosis. Morphological changes caused by EGFP-MVaf1 expression were characterized by fluorescence microscopy, in Z-stack acquisition mode (Figure 2a). EGFP-expressing control cells exhibited flattened shapes, stress fibers, and normal nuclear size and shape. Conversely, cells expressing EGFP-MVaf1 were round, exhibited plasma membrane blebs, a peripheral F-actin ring, as well as lack of stress fibers and loss of contact with the substrate. After $20 \mathrm{~h}$ of transfection, $\sim 70 \%$ of cells expressing EGFP-MVaf1 exhibited pyknotic nuclei. Time-lapse microscopy of cells expressing EGFP-MVaf1 provided images of membrane blebbing, followed by cell condensation and fragmentation into apoptotic bodies in a 10-min period (Figure $2 \mathrm{~b}$ and Supplementary video). These morphological features were not observed in EGFP control cells (Figure $2 \mathrm{~b}$ and Supplementary video). For a detailed analysis of cell surface, we performed scanning electron microscopy (SEM) of EGFP-MVaf1-transfected cells on Cell Locate coverslips (Figure 2c). Figure 2c and Supplementary video show pair of cells in an advanced stage of apoptosis, undergoing a budding process, followed by separation of cell fragments into apoptotic bodies. DNA fragmentation was also assessed by TUNEL staining (Figure 2d). Representative phase contrast and red fluorescence images show that $36.6 \%$ of cells expressing EGFP-MVaf1 were TUNEL positive (red), in contrast to $6.9 \%$ of EGFP-expressing cells, $48 \mathrm{~h}$ post transfection.

MVaf1 binds to DLCs and co-localizes with DLC2. To gain further mechanistic insights into the observed induction of apoptosis, we assessed the ability of MVaf1 or MVcf to interact with DLCs. Pull-down assays were performed using bacterially expressed and purified proteins fused to either glutathione-S-transferase (GST) or maltose-binding protein (MBP) (Figure 3a). Immobilized GST-MVaf1, like full-length myosin-Va MT (GST-MT) exhibited binding to MBP-DLC2, whereas GST-MVcf did not. Also, immobilized GST-MVaf1 and GST-MT captured EGFP-DLC1 or EGFP-DLC2 from HEK293 cell lysates (Figure 3b). Confocal microscopy of cells transfected with either EGFP-DLC2 or DsRed-MVaf1 showed that fusion protein is usually distributed throughout the cell (Figure 3c, top panel). In co-transfected cells, fusion proteins co-localized ( $92 \%$ index) and were mostly retained in the cytoplasm (central and bottom panels). Next, we aimed to minimize MVaf1-induced cell death by co-expressing DLC2. Co-expression of DLC2 protected against the effect of MVaf1 (Figure 3d). These observations are indirect evidence that MVaf1 exerts its apoptotic effect by sequestering DLC2.

MVaf1 and full-length myosin-Va medial tail sequester DLCs, but are unable to co-capture Bmf. Based on evidence that DLC2 works as an adaptor protein between myosin-Va and Bmf, we investigated the ability of MVaf1 to form a trimeric complex with DLC2 and Bmf using pull-down assays (Figures $3 e-g$ ). Firstly, we found that GST-DLC2 binds to Bmf with a far greater affinity than GST-DLC1 (Figure $3 e$ ) and showed that DLC2 can co-capture EGFPBmf and EGFP-Bcl2 present in cell lysates from HEK-293T (Figure 3f). Taken together, these data suggest the formation of a DLC2-Bmf-Bcl2 complexes, confirming previous evidence of this trimeric complex formation, ${ }^{10}$ and that Bmf binds DLC2 selectively. ${ }^{16}$ Next, EGFP-DLC2 or EGFP-DLC1 were co-expressed with EGFP-Bmf in HEK293T cells, and the soluble extracts were incubated with glutathione resin containing immobilized GST-MT or GST-MVaf1. Although GST-MVaf1 and GST-MT unequivocally bound EGFP-DLC1 and EGFP-DLC2, Bmf remained in solution (Figure $3 \mathrm{~g}$ ). This indicates DLC2 did not function as an adapter protein between MVaf and Bmf. Also, preliminary assays (data not shown) suggest that co-expression of MVaf1 with DLC2 and Bmf halted the formation of the trimolecular complex between DLC2, Bmf, and immobilized GST-Bcl2. These data suggest that Bmf binds to $\mathrm{Bcl} 2$, but DLC2 remains in the soluble fraction together with MVaf1.

Multiple cell lines are sensitive to MVaf1-triggered cell death and may be influenced by myosin-Va/DLC2 levels. As in B16-F10 cells, EGFP-MVaf1-induced cell death in two human melanoma cell lines, WM35 and WM902 (Figure 4a). 
a

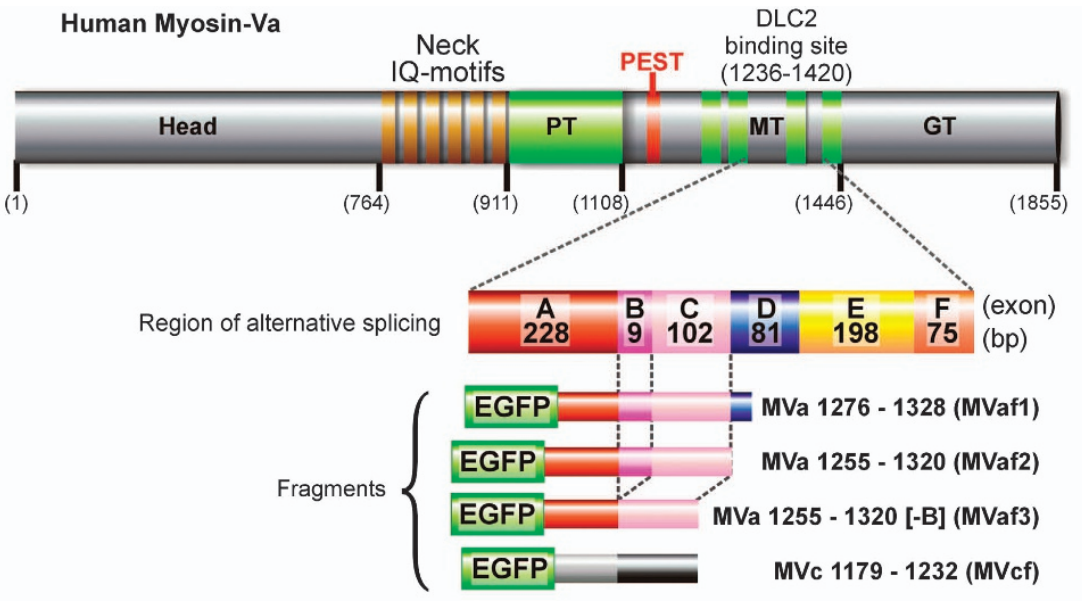

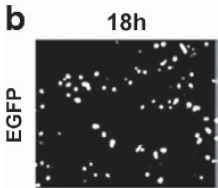

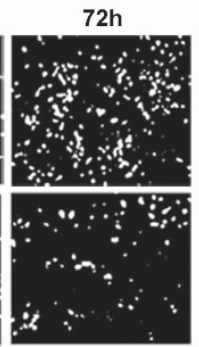

d
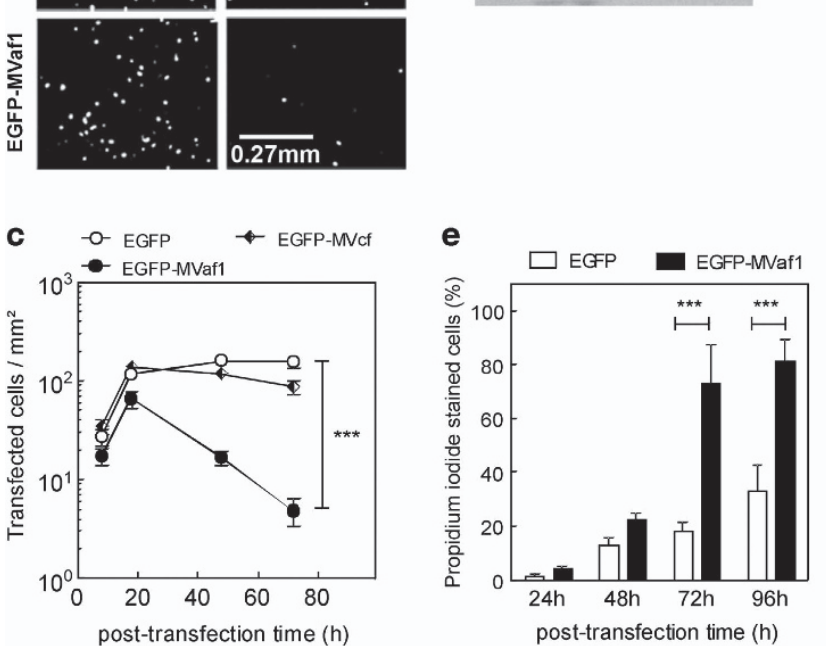

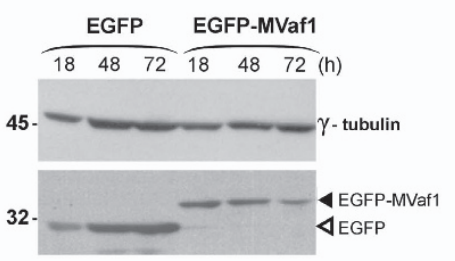

f

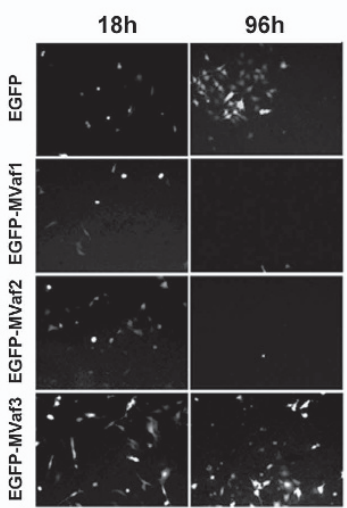

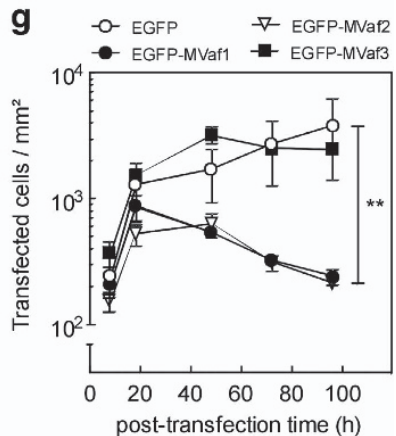

Figure 1 MVaf expression reduces melanoma cell viability in an exon B-dependent manner. (a) Schematic diagram representing the primary structure of myosin-Va heavy chain shows the positions of the structure sub-domains (H, head; N, neck; PT, proximal tail; $M T$, medial tail; GT, globular tail) and the DLC2 binding site in the MT domain, with exon combination A, B, C, D, E and F. Exons B, D, and F are subject to alternative splicing. Detail: schematic diagram of selected segments for recombinant constructs. EGFP-MYO5A ${ }^{(1276-1328[+B])}$ fragment (MVaf1), and variants, MVaf2 ${ }^{(1255-1320[+B])}$ and MVaf3 ${ }^{(1255-1320[-B])}$. EGFP-MYO5C ${ }^{(1179-1232)}$ construct was used as a control and comprised a region with low sequence conservation between the two paralogs (myosin- $\mathrm{Va}$ and $-\mathrm{Vc}$ ). (b) Representative images of B16-F10 cell cultures, 18 and $72 \mathrm{~h}$ post transfection with pEGFP, pEGFP-MVcf and pEGFP-MVaf1. Images were acquired under a fluorescence stereomicroscope. (c) Proliferation rates were determined as the average number of fluorescent cells per area of growth $\left(\mathrm{mm}^{2}\right)$ after $8,18,48$ and $72 \mathrm{~h}$ post transfection. ${ }^{* *}$ Statistic significance $(P<0.001)$. Kinetics of recombinant protein expression levels: (d) EFGP expression in B16-F10 cell lysates was quantified through ImageJ software analysis of western blots generated with an anti-EGFP (bottom panel) relative to $\gamma$-tubulin, a loading control (top panel). (e) Viability of cells expressing EGFP-MVaf1 or EGFP was determined by PI staining of dead cells at indicated times post transfection. ${ }^{* *}$ Statistically significant mean difference between EGFP and EGFP-MVaf1 groups $(P<0.001)$. (f) Representative images of cultures taken 18 and $96 \mathrm{~h}$ post transfection with pEGFP (control), pEGFP-MVaf1, pEGFP-MVaf2 or pEGFP-MVaf3. (g) Proliferation rates of B16-F10 cell cultures expressing either EGFP (control), EGFPMVaf1, EGFP-MVaf2 or EGFP-MVaf3 were determined as the average number of fluorescent cells per area of growth ( $\left.\mathrm{mm}^{2}\right)$, per dish $(n=3)$ at $8,18,48,72$ and $96 \mathrm{~h}$ post transfection. ${ }^{*}$ Statistically significant mean difference between control (EGFP) and test groups (EGFP-MVaf1 and EGFP-MVaf2) $(P<0.01)$

Population decline was delayed in WM902 (48 h), suggesting that this line is more resistant than WM35 to death induced by EGFP-MVaf1. Expression levels of MYO5A and DLC2 mRNAs were 1.4-fold and 2-fold, respectively, higher in
WM902 cells than in WM35 cells (Figure 4b), which can account for the relative resistance of WM902. Nonetheless, unrelated genetic alterations could account for this difference, as WM902 is derived from a tumor of a more advanced 
a
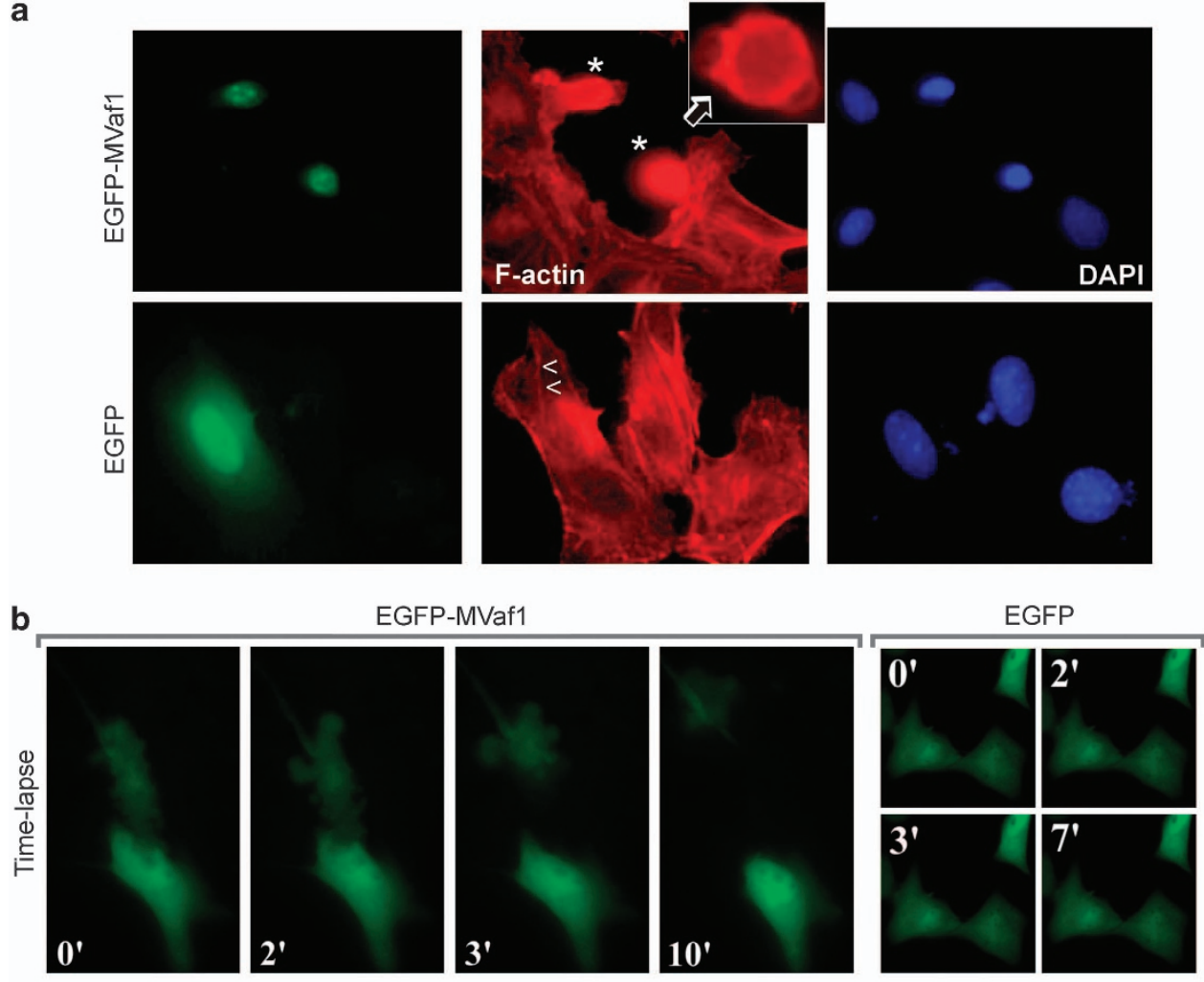

C
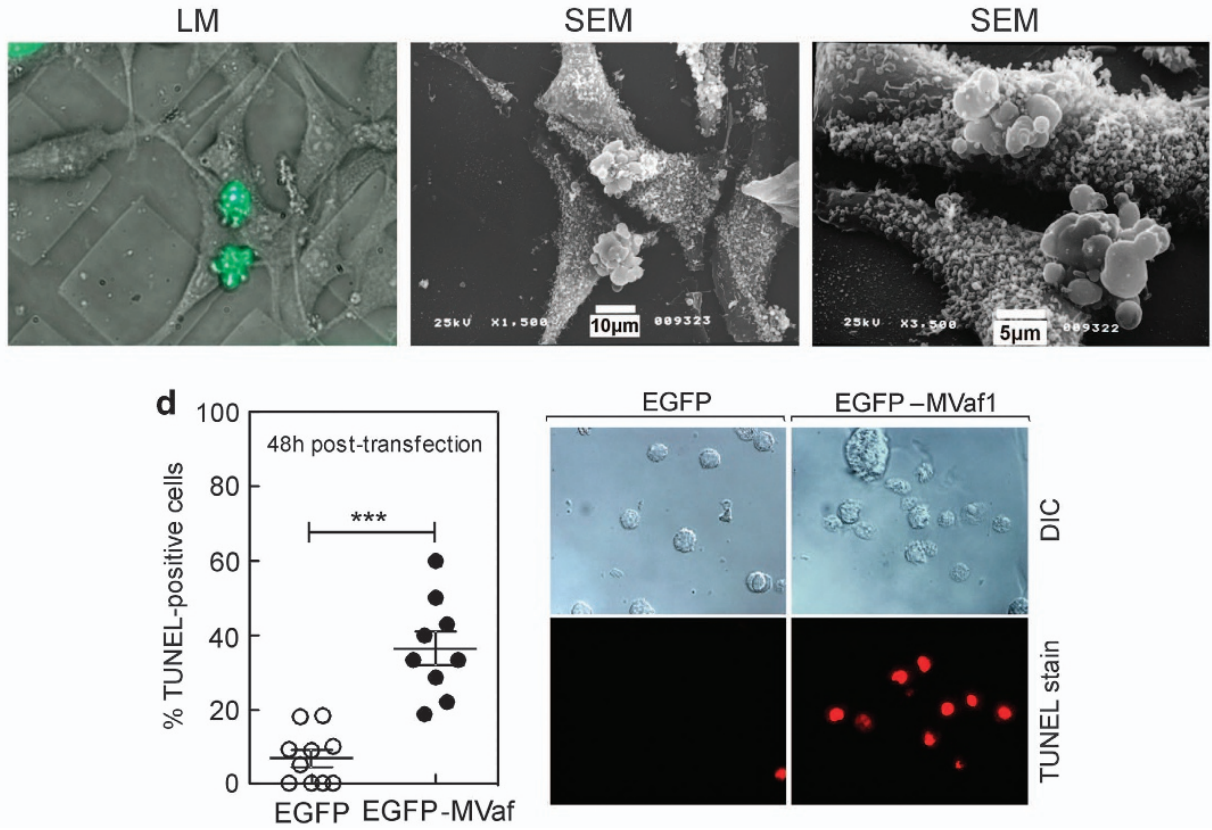

EGFP-MVaf1

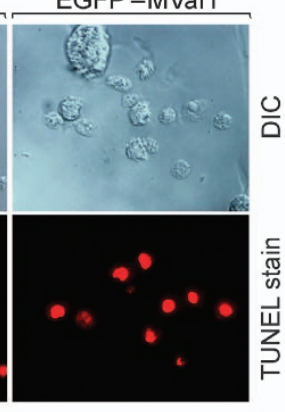

Figure 2 Cells expressing MVaf1 developed morphological features of apoptosis. Fluorescence and correlative light microscopy (LM)/SEM images are shown of B16-F10 cells, expressing either EGFP-MVaf1 or EGFP (control). (a) Fluorescence images show EGFP, F-actin (Phalloidin-Rhodamine) and nuclei (DAPI) staining. EGFP-MVaf1expressing cells $\left(^{*}\right)$ lack stress fibers, prominent in control cells $(>,<)$, and display a peripheral F-actin ring, as seen in the single section image (insert, arrow). (b) Time-lapse. B16-F10 cells grown on glass-bottom microwell dishes $(\Phi 35 \mathrm{~mm}$ ) and transfected with pEGFP-MVaf1 or pEGFP (control). (c) SEM images of B16-F10 cells transfected with pEGFP-MVaf1. B16-F10 cells grown on CELLocate-microgrid glass coverslips were imaged by LM, $24 \mathrm{~h}$ post transfection. SEM images of the corresponding cells were taken in two subsequent magnifications. Transfected cells exhibit apoptotic features typical of late execution phase, such as loss of attachment and plasma membrane blebs. (d) Apoptotic response to EGFP-MVaf1 involves DNA fragmentation. Detection of DNA fragmentation in suspended B16-F10 cells was assessed by the TUNEL assay. Scatter dot plot with mean \pm S.E.M. showing the ratio of TUNEL-positive nuclei to total nuclei counts in EGFP- and EGFP-MVaf1-expressing cultures $(n=10$ fields). Each data point represents a pair of images, i.e., one field. ${ }^{* \star}$ Statistically significant mean difference between EGFP and EGFP-MVaf1 groups $(P<0.001)$. Right panel shows representative differential interference contrast (DIC) and TUNEL stain (red) images of a single field from either EGFP and EGFP-MVaf1-expressing cultures 
a

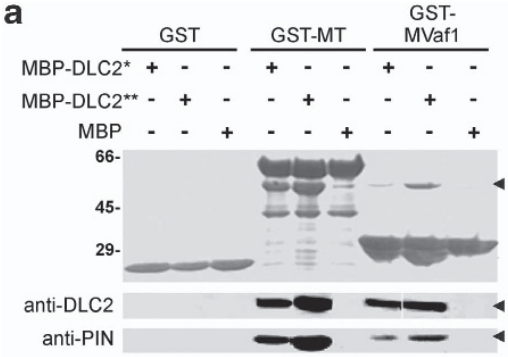

b

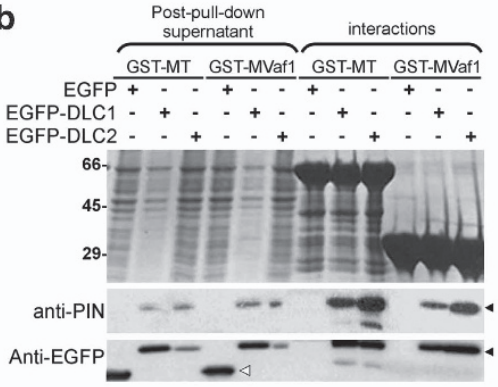

c

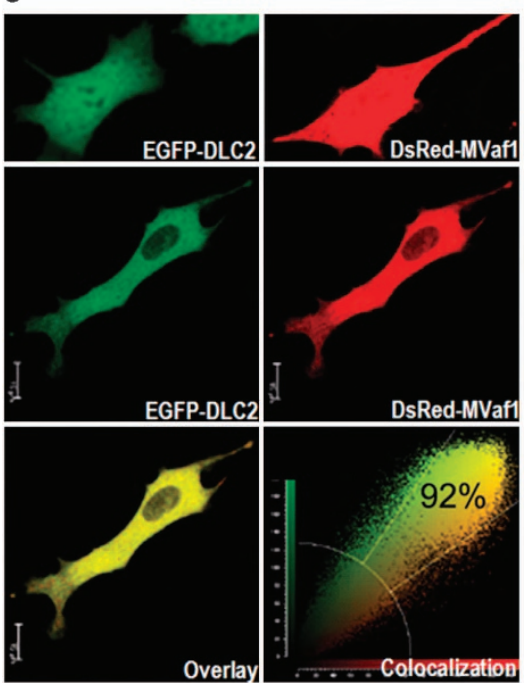

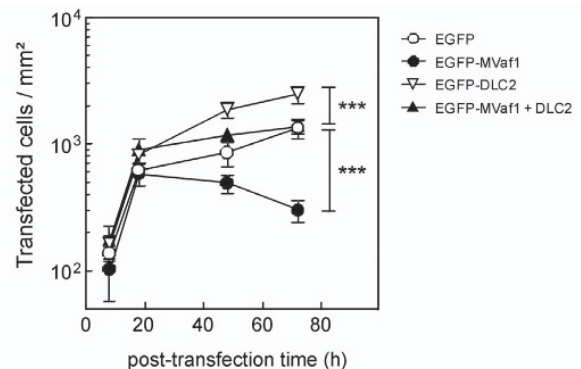

e

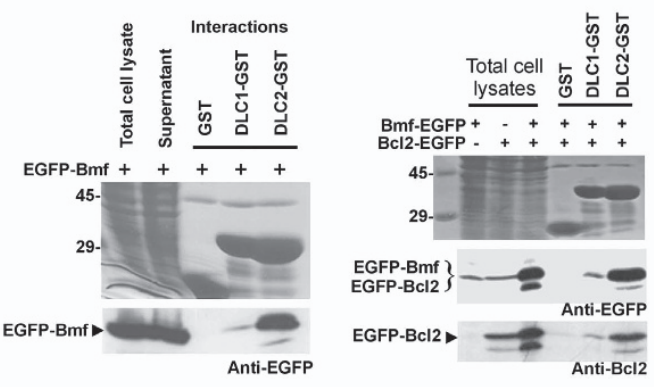

g

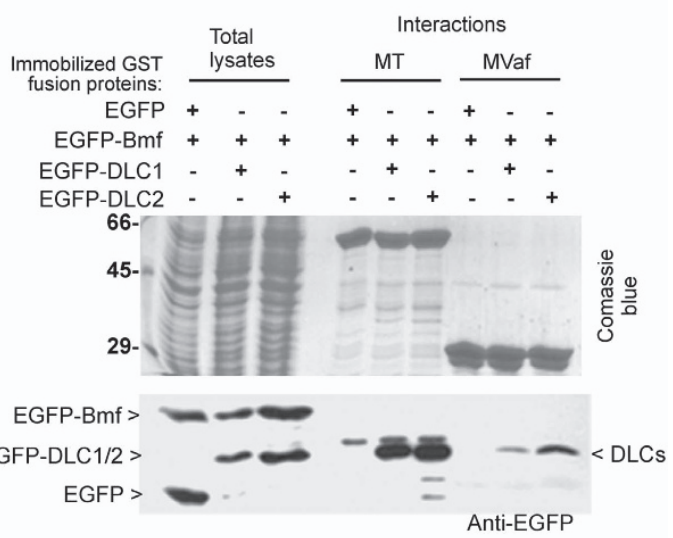

Figure 3 Protein interaction and co-expression assays. (a) MVaf1, but not MVcf, interacts with DLC1 and DLC2 in vitro. Bacterially expressed and purified GST or GST fusion proteins immobilized on Glutathione-Sepharose 4B beads were incubated with $200 \mathrm{ng}\left({ }^{*}\right)$ and $800 \mathrm{ng}\left({ }^{* *}\right)$ of purified MBP-DLC2 proteins or MBP alone. Full-length myosin-Va MT fused to GST (GST-MT) was used as a positive control for interaction with DLC2. The purified proteins were separated on 5-20\% SDS-PAGE gel and stained by Coomassie blue (top panel). The migration positions of the molecular mass markers $(\mathrm{kDa})$ are indicated on the left hand side. Pulled down proteins were blotted with antiDLC2 and anti-PIN antibodies (bottom panel). Black arrowheads indicate the migration position of MBP-DLC2. (b) GST pull-down experiments for MVaf1 and DLC1 or DLC2. Bacterially expressed and purified GST-MVaf1 and GST-MT fusion proteins immobilized on Glutathione-Sepharose 4B beads were incubated with cell lysates from HEK-293T, expressing either EGFP-DLC1, EGFP-DLC2 or EGFP alone. Proteins from pull-down interactions or post-pull-down supernatants were separated on 5-20\% SDS-PAGE gel and stained by Coomassie blue (top panel). Western blots were probed with anti-EGFP and anti-PIN antibodies (bottom panels). Black arrowheads indicates EGFP-DLC 1 or 2, and white arrowhead indicates EGFP. (c) DsRed-MVaf1 co-localizes with EGFP-DLC2 in the cell cytoplasm. B16-F10 cells expressing either (top panel), or both (central panel) EGFP-DLC2 and DsRed-MVaf1, were fixed and imaged by confocal microscopy. Colocalization was analyzed using Leica LAS-AF software. (d) Coexpression of DLC2 with MVaf1 attenuates MVaf1-induced cell death. Proliferation rates of B16-F10 cell cultures expressing either EGFP (control), EGFP-MVaf1, EGFPDLC2 or co-expressing EGFP-MVaf1 and EGFP-DLC2 were determined as the average number of fluorescent cells per area of growth $\left(\mathrm{mm}^{2}\right)$, per dish $(n=4)$, after $8,18,48$ and $72 \mathrm{~h}$ post transfection. (e-g) GST pull-down experiments for investigating the interactions between MVaf1, DLCs, Bmf and Bcl2. Bacterially expressed and purified GST fusion proteins (as indicated) were immobilized on Glutathione-sepharose 4B beads and incubated with cell lysates from HEK-293T expressing EGFP-Bmf alone (e), or the combination of EGFP-Bmf plus EGFP-Bcl2 (f), or EGFP-Bmf plus either EGFP-DLC1 or EGFP-DLC2 (g). Proteins from pull-down interactions were separated on 5-20\% SDS-PAGE gel and stained by Coomassie blue (top panels). Western blots were probed with anti-EGFP (bottom panels) or anti-Bcl2, as indicated. Note strong interaction of Bmf with DLC2 and only a poor interaction with DLC1. Also note that immobilized DLC2 bound Bmf/Bcl2, whereas immobilized myosin-Va tail fragments pulled-down DLCs but left behind Bmf in the supernatant, indicating that DLC2 was unable to play an adaptor function

stage (vertical growth phase) than WM35 (radial growth phase), and cancer cells accumulate a diverse array of genetic mutations. Subsequently, we asked how sensitive murine Cloudman S91 melanoma cells (derived from dilute viral mouse) would be to apoptosis triggered by EGFP-MVaf1, considering that this cell line is virtually free of myosin-Va 
a

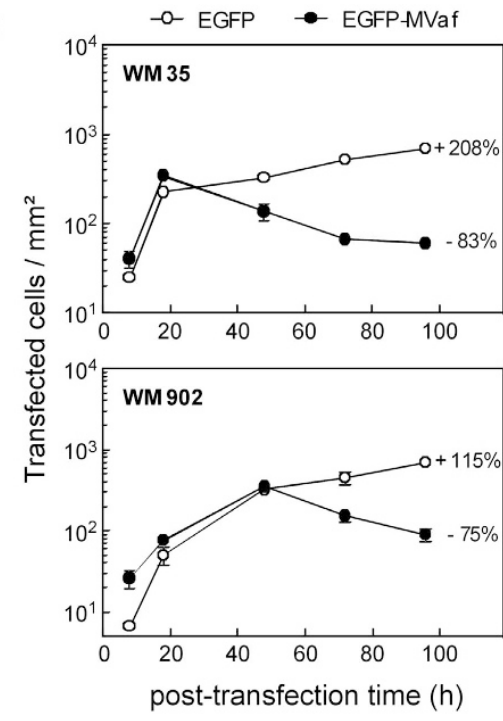

c
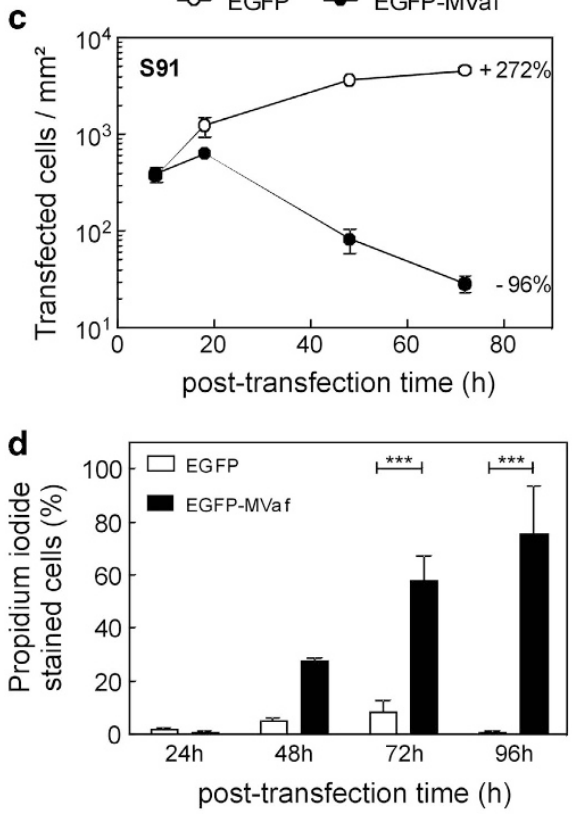

b
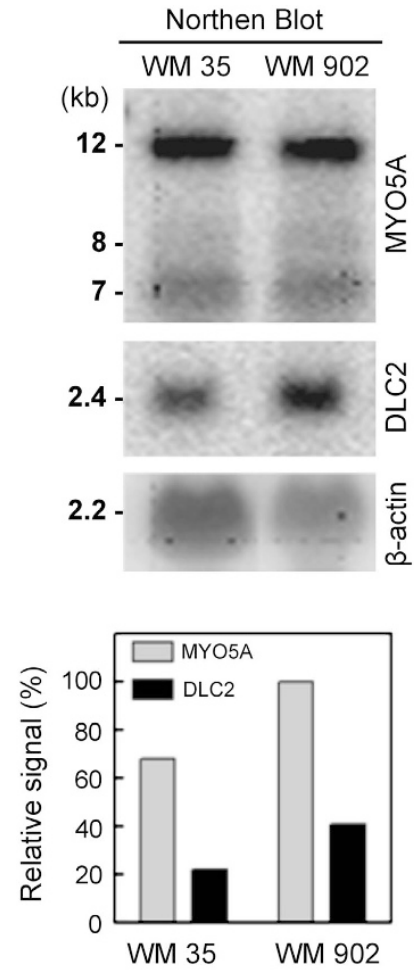

e

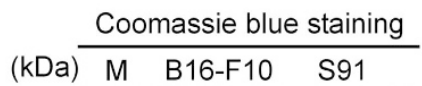

(CaM) 20-

(DLC 1/2) 10 -
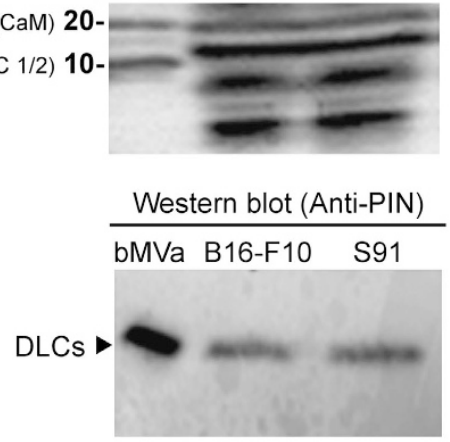

Figure 4 Human melanoma cell lines are prone to cell death triggered by MVaf1 and levels of myosin-Va/DLC2 appears to influence cell death sensitivity. (a). Proliferation rates of WM35 and WM902 cells expressing either EGFP (control) or EGFP-MVaf1 were determined as the average number of fluorescent cells per area of growth (20 random fields of $1.6 \mathrm{~mm}^{2}$ per dish; $n=4$ ), after 8,18,48,72 and $96 \mathrm{~h}$ of transfection. Rates are expressed as a percentage increase or decrease in culture proliferation from 18-96 $\mathrm{h}$ (WM35) or 48-96 h (WM902) post transfection (on the right-hand side of proliferation curves). (b) Northern blot analysis. MYO5A and DLC2 gene expression profiles in human melanoma cell lines WM35 and WM902. Densitometry of the specific bands was done measured by the ratio of pixel intensity (relative signal) using ImageJ gel analysis software; $\beta$-actin mRNA was used as the loading control. (c) Proliferation rate of Cloudman S91 mouse melanoma cells (bearing a MYO5A loss-of-function mutation) expressing either EGFP (control) or EGFP-MVaf1. Rates expressed as percentage increase or decrease in proliferation from 18 to $72 \mathrm{~h}$ post transfection are indicated. (d) Cell death rates for Cloudman S91 cells expressing EGFP-MVaf1 and EGFP were determined by PI staining at 24, 48, 72 and $96 \mathrm{~h}$ post transfection. After imaging 20 random fields per dish $(n=5)$, the percentage of unviable cells was calculated relative to the total number of green fluorescent cells. (e) Detection of DLCs in B16-F10 and S91 cell lysates. Cell lysates from B16-F10 and Cloudman S91 were collected, equivalent amounts of total protein was loaded and analyzed by electrophoresis on a 5-20\% SDS-PAGE, stained with Coomassie blue (top panel). Anti-DLC/PIN, which recognizes both DLC 1 and 2, was used as detection antibody (bottom panel). bMVa, purified fraction of native myosin-Va from chick brain, was used as positive control for the detection of the light chain DLC2 (as indicated)

expression. The number of EGFP-MVaf1-expressing cells decayed very rapidly from 18 to $96 \mathrm{~h}$, such that cultures remained with only $5 \%$ of cells initially scored at $18 \mathrm{~h}$ (Figure 4c). Pl staining confirmed intense cell death (Figure 4d). The levels of DLCs were equivalent between
S91 and B16 cells (Figure 4e), indicating that the higher sensitivity of S91 to EGFP-MVaf1-induced death was not due to reduced DLC1/2 levels. We hypothesize that trapping of DLCs by MVaf1 is more effective because MVaf1 is not counteracted by the endogenous pro-survival myosin-Va in S91 
cells. In addition, this result implies that DLC2 probably also functions to promote cell survival independently of myosin-Va.

\section{MVaf-induced apoptosis is associated with cytochrome- $c$ and Smac release as well as caspase-9/-3 activation.} To evaluate whether EGFP-MVaf1 triggers apoptosis through the intrinsic pathway by inducing mitochondrial outer membrane permeabilization (MOMP), we investigated the occurrence of cytochrome- $c$ release. The number of cells with a diffuse cytochrome- $c$ staining pattern was higher among EGFP-MVaf1-expressing cells than among EGFP control or non-transfected neighbors. Diffuse cytochrome-c pattern increased from 14 to $41.4 \%$ in the 24-33h interval post transfection with EGFP-MVaf1, whereas reached only $8.6 \%$ rates in EGFP cells (Figure $5 a$ ). Subsequently, we monitored MOMP by Smac-Cherry release using time-lapse microscopy in cells co-expressing EGFP-MVaf1 and SmacCherry (Figure 5b and Supplementary video). EGFP-MVaf1 was intense and distributed throughout the cell, whereas Smac-Cherry changed from compartmentalized in mitochondria (punctate labeling) to a diffuse staining pattern. Soon after, cells exhibited characteristic features of apoptosis, such as membrane blebbing, loss of adhesion, and nuclear condensation, which culminated in fading of fluorescence. Caspase- 9 activation was involved in the apoptotic response triggered by EGFP-MVaf1, as the cleaved form of caspase-9 (37-kDa band) was predominant and the full-length form was less pronounced in lysates of cells expressing EGFP-MVaf1 than in control cell lysates (Figure $5 c$ ). The signal intensity ratio between active caspase- and pro-caspase- 9 was about sixfold higher in EGFP-MVaf1-expressing cells. To determine caspase-3 activation, we utilized a caged fluorochrome conjugated to caspase-3 substrate (Figure $5 \mathrm{~d}$ and Supplementary video). EGFP-MVaf1-expressing cells turned bright red fluorescent, denoting a sudden activation of caspase-3. This was immediately followed by plasma membrane blebbing cellular fragmentation and plasma membrane rupture, accompanied by an accentuated drop in green fluorescence. The whole process was completed in about 2 minutes.

Bmf/Bim and Bax/Bak are important but ultimately not essential for the apoptosis triggered by MVaf expression. To evaluate whether Bim/Bmf or Bax/Bak are required for the apoptotic response triggered by EGFP. MVaf1, we compared rates of apoptosis in wild-type (WT) murine embryonic fibroblasts (MEF ${ }^{\mathrm{WT}}$ ) with $\mathrm{Bmf} / \mathrm{Bim}$ or Bax/Bak double-knockout MEFs by scoring dead cells through video microscopy. Figure 5e represents the percentage of dead cells. MEF ${ }^{\mathrm{WT}}$ showed the highest scores of cell death (90\%). Conversely, double-knockout strains were less sensitive to induced cell death, with scores of $45 \%$ for $\mathrm{MEFs}^{\mathrm{Bim}-l-, \mathrm{Bmf}-l-}$, and $30 \%$ for MEFs ${ }^{\mathrm{Bax}-l-\text {, Bak }-l-}$ We also assessed the occurrence of Smac release in MEFs ${ }^{\mathrm{Bax}-1-, \mathrm{Bak}-1-}$, co-expressing EGFP-MVaf1 and Smac-Cherry. It was observed that cells at $31 \mathrm{~h}$ post transfection were still exhibiting a punctate Smac-Cherry labeling, although displaying morphological features of apoptosis (Figure $5 \mathrm{f}$ ). MEF ${ }^{\mathrm{WT}}$ underwent MOMP similar to B16-F10 melanoma cells (not shown).
MVaf1 expression hinders growth of B16-F10 solid tumors in mice. To assess the effect of MVaf1 expression on tumor growth we used a xenograft murine model. Tumors expressing EGFP appeared earlier and grew faster than tumors expressing EGFP-MVaf1, regardless of whether mice were treated with doxycycline or not (Figures $6 a$ and $b$ ). With regard to tumors carrying EGFP-MVaf1, we observed that in animals not receiving doxycycline tumors developed earlier and were larger than in treated animals. Nevertheless, in the absence of doxycycline, EGFP-MVaf1 tumors did not grow comparably to controls, possibly due to expression leakage, which was confirmed by western blots of solid tumor lysates (Figure 6c). Sorted EGFP-MVaf1 cells and EGFP control consisted of mixed populations, not clonal lineages, making unlikely that genomic integration could account for these results, as genetic alterations leading to unfavorable growth are not prone to be selected for in the population. Next, survival rates of mice injected with parental cells were compared with those injected with two distinct strains of EGFP-MVaf1-transduced cells, which were derived from single cell-isolated clones C3 and G3 (Figure 6d). Mice expressing EGFP-MVaf1 survived longer than control. In addition, we evaluated whether EGFP-MVaf1 would influence/susceptibility to conventional chemotherapeutic drugs (Supplementary Figure S1-F). After treatment with paclitaxel or etoposide, EGFP-MVaf1-expressing cells displayed 54 and 39\% more apoptotic cells than controls (Supplementary Figure S1-F), suggesting that EGFP-MVaf1 expression sensitizes cells to chemotherapy. Western blots show the levels of EGFP-MVaf1 expression in the cultures analyzed (Supplementary Figure S1-G). Finally, clonogenic assays with stably transduced B16-F10 cells (Supplementary Figure $\mathrm{S} 1-\mathrm{H}$ ) corroborated the notion that expression of MVaf1 restores susceptibility to apoptosis.

\section{Discussion}

Despite the suggested involvement of myosin-Va/DLC2 motor complex in anoikis-induced cell death, its roles in cancer remain largely unexplored. Resistance to anoikis leads to anchorage-independent growth and malignant transformation. ${ }^{12-14,17}$ In this work, we initially aimed at cloning the less conserved regions among the three myosin$V$ paralogs for functional studies. It was soon apparent that the expression of EGFP-MVaf1 led to a dramatic decline in the number of transfected cells due to apoptosis induction, as suggested by morphological features ${ }^{18}$ and molecular events typical of apoptosis. Release of cytochrome-c and Smac supports the involvement of MOMP, with subsequent activation of caspase-9/-3, in the MVaf1-induced apoptotic response. However, EGFP-MVaf1 did not seem to localize to mitochondria, suggesting that MOMP was an indirect consequence of MVaf1 action. Accordingly, we found that MVaf1 binds DLC2, which potentially impedes the docking of Bmf onto the actin cytoskeleton. This is further supported by the fact that MEFs lacking Bim/Bmf or Bax/Bak were less susceptible to induced cell death than MEF ${ }^{\mathrm{WT}}$, suggesting the requirement of these pro-apoptotic proteins as mediators of MVaf-induced MOMP. Attenuation of apoptosis in MEFs lacking Bmf/Bim suggests a mechanistic interaction similar 
to the one previously proposed by Puthalakat and co-workers. ${ }^{10,11}$ Nevertheless, Smac remained compartmentalized in dying MEFs ${ }^{\mathrm{Bax}-/-, \mathrm{Bak}-/-}$ and apoptosis was not completely abolished in MEFs lacking Bim/Bmf, indicating that high expression of MVaf1 triggers cell death through multiple intracellular mediators and pathways.

Remarkably, Bmf sequestration to the actin cytoskeleton through DLC2 was reported to be involved with resistance to MEK inhibitors in melanoma cells, so that overexpression of Bmf in resistant cells does not induce apoptosis, whereas mutant BmfA69P, with decreased DLC2 binding, promotes cell death. ${ }^{19}$ In addition, acquired resistance of human melanoma cells toward the oncogenic BRAF inhibitor PLX4032 (vemurafenib) was associated with epigenetic silencing of $\mathrm{Bim}$ and $\mathrm{Bmf}^{20}$ Consistently, we demonstrated herein that MVaf1 expression hindered melanoma growth and increased survival in mice. Furthermore, we demonstrated that EGFP-MVaf1 expression
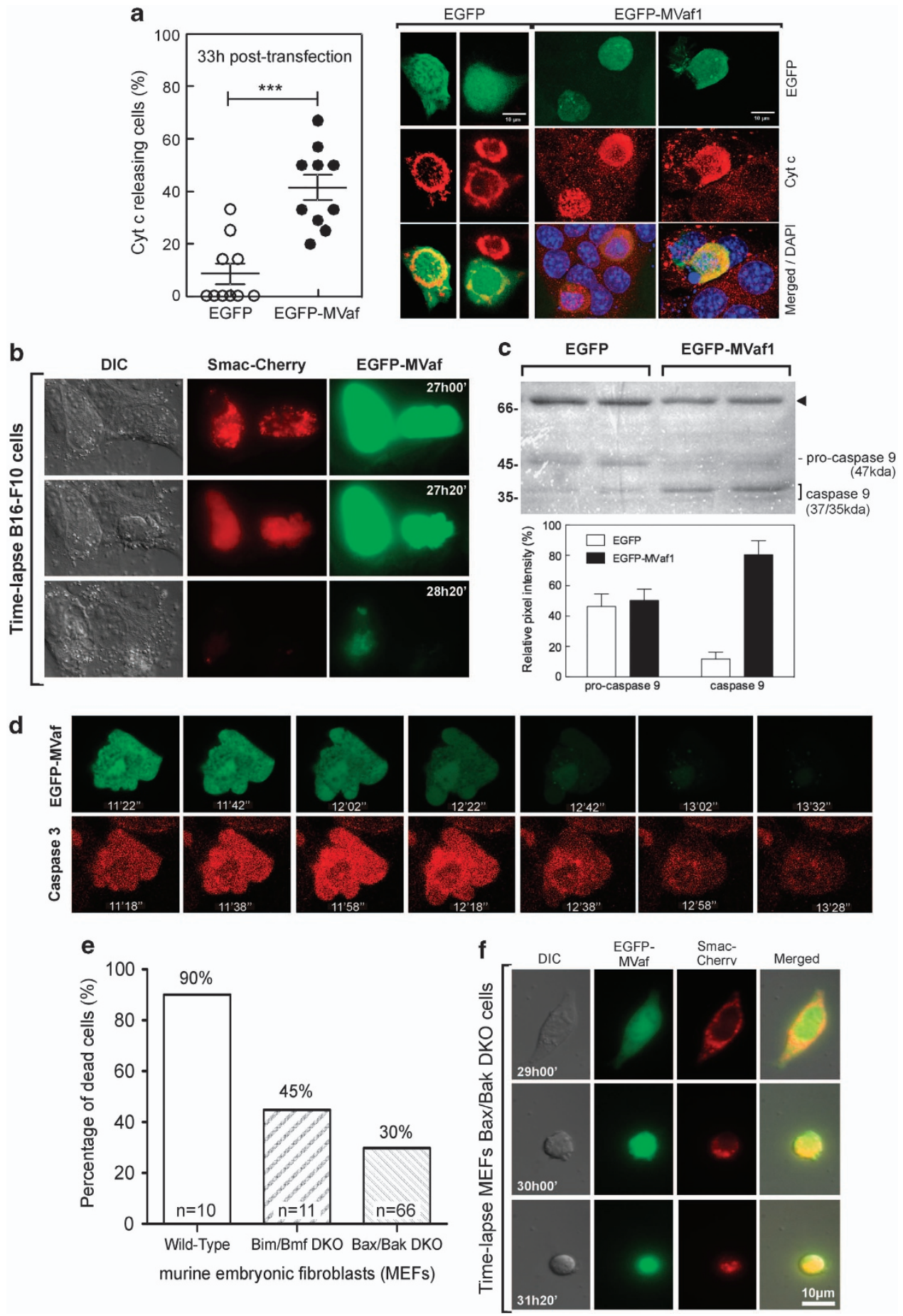
sensitizes B16-F10 cells to paclitaxel and etoposide, suggesting that MVaf1 could have a significant role as a co-adjuvant against chemoresistant cells.
Interestingly, emerging evidence implicates myosin-Va in cell survival and cancer-promoting functions. Myosin-Va was found in a complex with $\mathrm{Bcl}-\mathrm{xL}$, which has been suggested to a

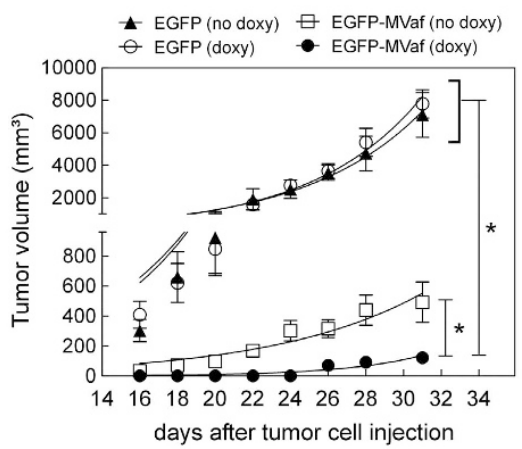

b

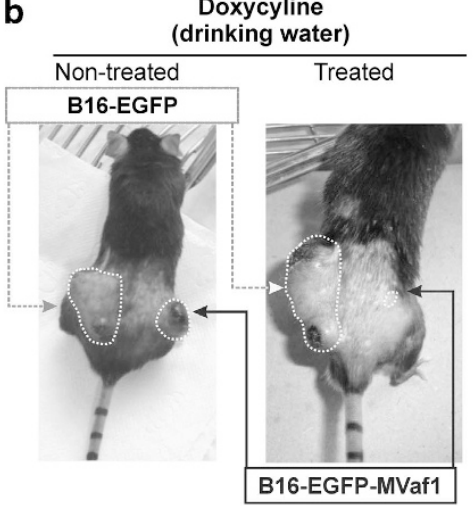

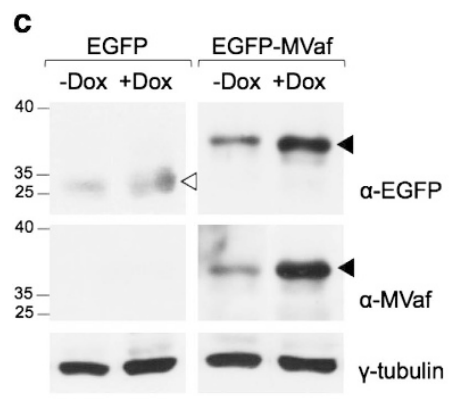

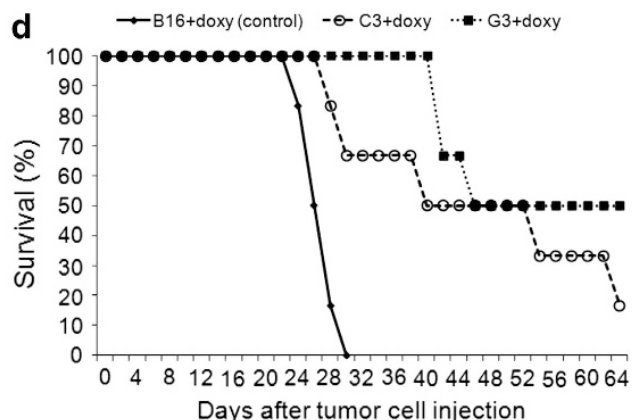

Figure 6 Effects of MVaf1 expression on tumor growth and animal survival rate in the B16-F10-bearing murine model. (a) Tumor growth rates. Mice bearing EGFP-MVaf1expressing cells and treated with doxycycline (doxy) did not develop tumors until after the 24-day observation period. Significant differences were verified using an unpaired two-tailed Student's t-test. $\left(^{*}\right) P<0.05$ compared with controls, as indicated on the figure. (b) Representative images of mice bearing tumors of EGFP-MVaf1 and EGFPtransduced B16-F10 cells. The B16-F10 melanoma tumor incidence rate and size were dramatically decreased by EGFP-MVaf ( \pm doxy). Arrows show the site for EGFPMVaf1-expressing tumor (black) and EGFP-expressing tumor (gray) ( $n=8 /$ group). (c) Western blots of solid tumor lysates. Lysates were prepared 30 days post inoculation and were probed with commercial anti-GFP antibody ( $\alpha$-EGFP) and laboratory-made anti-MVaf ( $\alpha$-MVaf); $\gamma$-tubulin was used as loading control. Black arrowheads indicate EGFP-MVaf1 and white arrowhead indicates EGFP. Analysis shown in a-c were done with whole populations of EGFP-MVaf or EGFP-transduced cells. (d) Survival rates of mice bearing subcutaneous tumors of parental B16-F10 cells or derived clones (C3 and G3) expressing EGFP-MVaf. The survival rates and survival time increased in both $\mathrm{C} 3+$ doxy and G3 + doxy groups ( $n=6$, in observation time point: 66 days post inoculation) compared with the B16 + doxy control group (no animal survived beyond 30 days). $\mathrm{C} 3$ and G3 are single-cell clones of EGFP-MVaf1 derived from transduced B16-F10 cell

Figure 5 Cells expressing MVaf1 undergo mitochondria-mediated apoptosis. (a) Immunolocalization of Cyt-c. B16-F10 cells expressing either EGFP-MVaf1 or EGFP (control) were stained by immunocytochemistry with anti-Cyt-c antibody. Cells displaying a diffuse cytosolic Cyt-c staining were counted among 10 random fields per coverslip, and the percentage of cells with released Cyt- $c$ was calculated relative to the total number of green fluorescent cells. Right panel shows representative images of cells, expressing either EGFP or EGFP-MVaf1, $33 \mathrm{~h}$ post transfection. In most cells expressing EGFP (control), Cyt-c staining showed an abundant and granular perinuclear pattern indicating mitochondrial compartmentalization. EGFP-MVaf1-expressing cells showed more diffuse red fluorescence pattern indicating their release into cytosol (scale bar: $10 \mu \mathrm{m}$ ). (b) Smac-Cherry time-lapse. B16-F10 cells were co-transfected with pEGFP-MVaf1 and pSmac-Cherry. In the example shown, at $27 \mathrm{~h}$ post transfection, Smac-Cherry (red) is still compartmentalized in mitochondria. At $27 \mathrm{~h}$ and $20 \mathrm{~min}$, Smac-cherry displays a widespread distribution within the cell. At $28 \mathrm{~h}$, cells lost their fluorescence. (c) Western blot for caspase-9. Cell lysates from B16-F10 cells expressing either EGFP-MVaf1 or EGFP (control) were collected at 24, 36, 48 and $60 \mathrm{~h}$ post transfection, combined and analyzed by western blotting with anti-caspase-9, as detection antibody. Duplicates were run for each sample. (c, top) Bands corresponding to pro-caspase-9 $(47 \mathrm{kDa})$ and caspase-9 $(37 \mathrm{kDa})$, were identified in all samples; black arrowhead indicates a nonspecific cross-reacting band picked up by caspase-9 antibody, and served as loading control. (c, bottom) Graphical representation of the relative intensity of pro-caspase- and caspase- 9 bands measured by the ratio of pixel intensity using ImageJ gel analysis software. (d) Caspase-3 activation. Frames selected from video acquired by time-lapse confocal microscopy. At $24 \mathrm{~h}$ post transfection with pEGFP-MVaf1 or pEGFP (control), B16-F10 cells were treated with caspase-3 Fluorogenic Substrate IX (Calbiochem) and (Z-DEVD)2-Rh110 for 10 min in order to monitor caspase-3 activity.

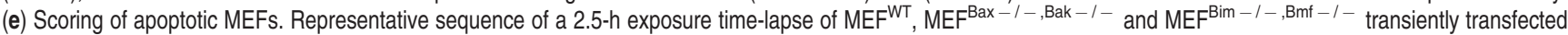
with pEGFP-MVaf1 and imaged $24 \mathrm{~h}$ later. Data is represented as percentage of dead cells relative to the total number of green fluorescent cells. Ten independent time-lapse

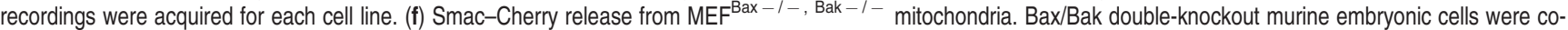
transfected with pEGFP-MVaf1 and pSmac-Cherry. Images represent excerpts from DIC and fluorescence microscopy videos of a representative cell expressing EGFPMVaf1. In the example shown, at $31 \mathrm{~h}$ and 20 min post transfection, Smac-Cherry (red) is still compartmentalized in mitochondria of the dying cell (observed as a punctate staining pattern). Wild-type MEFs underwent permeabilization of the mitochondrial outer membrane similar to B16-F10 melanoma cells (not shown). So MOMP is involved but ultimately does not appear to be crucial for the occurrence of cell death 
promote aggressive behavior of islet tumor cells. ${ }^{21}$ Myosin-Va was proposed as a useful biomarker of invasiveness for nonfunctioning pituitary adenomas, ${ }^{22}$ and upregulation of myosinVa by Snail has been correlated with cancer metastasis. ${ }^{23}$ Other intriguing findings involving myosin-Va could as well contribute to death resistance and other hallmarks of cancer. For instance, myosin-Va was involved in the transport of PTEN to the plasma membrane, thereby modulating PI3K pathway neuronal cells, ${ }^{24}$ in plasma membrane receptor recycling, ${ }^{25}$ and trafficking of the glucose transporter GLUT4 in adipocytes. ${ }^{26}$ Concurrently, GLUT4 has been claimed to function in cancer cells. ${ }^{27}$ Myosin-Va was also shown to localize to the centrosome and mitotic spindle, ${ }^{28}$ and to nuclear speckles, ${ }^{29}$ which may be linked to a putative role in the transport of mRNA. ${ }^{30}$ Interestingly, recent data from our group reveal that in comparison to melanocytes, various melanoma cell lines show strong upregulation of myosin-Va, and its depletion with shRNAs attenuates anchorage-independent growth and invasion in vitro (Alves et al., manuscript submitted), compatible with the notion that myosin-Va contributes to anoikis resistance. Here, we found that $\mathrm{S} 91$ melanoma cells (virtually free of myosin-Va) were remarkably sensitive to EGFP-MVaf1-induced cell death. Consistently, S91 cells were previously reported to be surprisingly sensitive to anoikis, accounting for the poor metastatic ability of this cell line. ${ }^{17}$ We understand that in the absence of endogenous myosin-Va to compete for DLC2, MVaf is more effective in sequestering the light chains and, consequently, unleashing the pro-apoptotic factors. On the other hand, this result denotes that DLC2 probably also functions to promote cell survival independently of myosin- $\mathrm{Va}$, by direct interaction with Bmf and/or additional partners. ${ }^{3}$

We demonstrated that MVaf1, which contains the core of DLC-binding site, binds both DLC2 and DLC1, but the complex MVaf1/DLC2 was unable to bind Bmf, suggesting that induction of apoptosis by MVaf1 is due to sequestration of DLC2, and probably also DLC1. As a result, both proapoptotic molecules, namely Bmf and Bim, would be unleashed and stabilized in the cytosol, allowing interaction with mitochondrial anti-apoptotic Bcl2 family members and consequent launch of apoptosis, as proposed in our schematic model (Figure 7). Also, Bim may directly activate Bax/ Bak as reported previously. ${ }^{31,32}$ The hypothesis postulated here is strongly supported by previous observations ${ }^{10,11}$ and may possibly explain the intense apoptotic response to MVaf1, resulting from concomitant action of both proapoptotic proteins. These findings are equally corroborated by the dependence of MVaf1-induced apoptosis on the three amino acid residues DDK, encoded by exon $B$, which was shown to be required for DLC binding by Hódi et al. ${ }^{15}$ and Wagner et al. ${ }^{33}$ The postulated model also integrates several studies on pro-survival and pro-apoptotic kinase signaling pathways controlling Bim and Bmf activity, and attempts to explain how such mechanisms may contribute to the intense apoptotic response induced by MVaf1. Treatment of epithelial cells with epidermal growth factor (EGF) has been shown to protect against UV-induced apoptosis, by the activation of p21-activated kinase (PAK1). ${ }^{34}$ EGF-activated PAK1 phosphorylates both DLC1 and Bim, inducing their dissociation and preventing the function of Bim by targeting it for proteasomal degradation. Furthermore, PAK1 was also demonstrated to interact with DLC2. ${ }^{35}$ We hypothesize that overexpressed MVaf1 binds DLC1 and DLC2, preventing them from binding their respective partners Bim and Bmf. Consequently, both pro-apoptotic proteins would not be targeted for degradation. Instead, they would be available for the pro-apoptotic or stress-activated c-Jun N-terminal kinase (JNK) that phosphorylates unbound Bim and Bmf, stabilizing them by

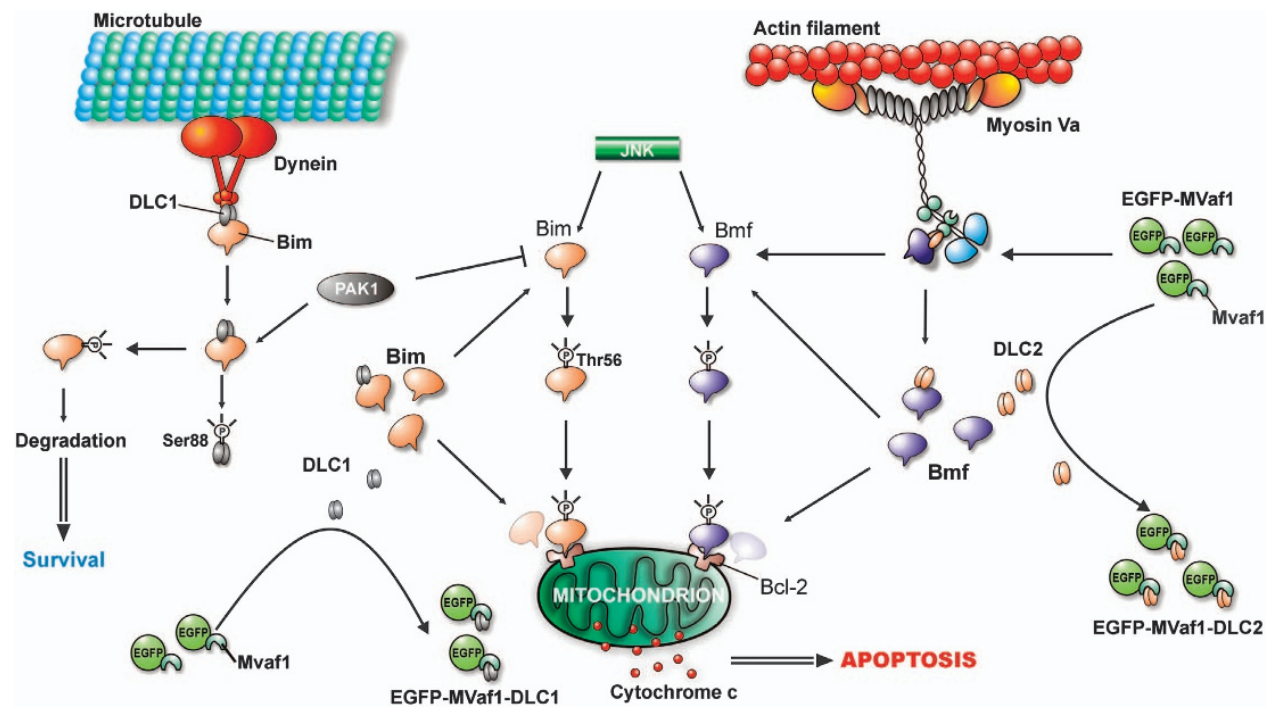

Figure 7 Hypothetical model of the mechanism by which MVaf1 overexpression induces apoptosis. It has been previously demonstrated that after apoptotic stimuli, EGFactivated PAK1 phosphorylates both DLC1 and Bim, inducing their dissociation and targeting the pro-apoptotic protein for degradation by the ubiquitin-proteasome system, thus promoting survival. ${ }^{31,32}$ Furthermore, PAK1 has also been demonstrated to interact with DLC2. ${ }^{33}$ Investigation of protein connections by pull-down assays suggests that upon high levels of expression, MVaf1 would sequester both DLC1 and DLC2, preventing them from binding their respective partners Bim and Bmf. Hence, both pro-apoptotic proteins would not be targeted for degradation by PAK1. Instead, they would be available for the pro-apoptotic or stress-activated JNK that phosphorylates unbound Bim and Bmf, preventing their ubiquitination. ${ }^{34,35}$ Stabilized Bmf, and possibly Bim, would finally trigger apoptosis via mitochondria-mediated pathway 
preventing their ubiquitination. ${ }^{36} \mathrm{Bim}$ and $\mathrm{Bmf}$ were shown to be essential for anoikis and suppression of Bmf by oncogenes was required for transformation of MCF10A mammary cells. ${ }^{37}$ Similarly, Bim and Bmf mediate apoptosis in response to MEK inhibitors and sequestering of Bmf leads to drug resistance. ${ }^{19}$ Also, Bim and Bmf were found to act in concert to induce anoikis-like apoptosis in HeLa cells infected with Neisseria gonorrhoeae. ${ }^{38}$ Infection-induced loss of Bim and Bmf from the cytoskeleton fraction required the activation of JNK-1. Accordingly, depletion/inhibition of JNK-1, Bim or Bmf prevented the activation of Bax/Bak. Moreover, cooperation between Bmf and Bim in vivo was demonstrated. ${ }^{39}$ Altogether, these data support our hypothetical model.

Despite sharing 93\% sequence identity, it has been demonstrated that both DLCs show unambiguous specificity for their respective $\mathrm{BH} 3$-only ligands and motor complexes. ${ }^{16}$ Nevertheless, myosin-Va has identical binding characteristics to recombinant DLC1 or DLC2 in vitro. ${ }^{5}$ The contrast between in vivo-and in vitro-binding specificity suggests an intrinsic molecular mechanism or the involvement of other proteins for the specific compartmentalization of DLCs and their proapoptotic cargoes. ${ }^{16}$ Hence, it is possible that the small soluble recombinant fragment MVaf1 is not subjected to specific DLC-compartmentalization rules. Furthermore, the remarkable apoptotic effect triggered by MVaf raises a central question for future investigation: Is it possible that upon apoptotic stimuli, cells naturally produce a similar molecule? This would not be surprising, because many cellular functions are controlled by the formation of biologically active proteolytic fragments, ${ }^{40-43}$ and a precedent that supports this idea is that myosin-Va bears a calpain cleavage region, downstream of the strong PEST motif, in the medial tail. ${ }^{44-47}$

In conclusion, this work provides a proof of the concept that $\mathrm{Bmf}$ is kept in check by interaction with myosin-Va/DLC2, ${ }^{10}$ and disruption of this interaction leads to apoptosis. Yet, data shown here suggest that further studies are needed to clarify the exact role for myosin- $\mathrm{Va}$ in this process. In any case, our results reinforce previous findings ${ }^{19,34,37}$ that the restraining mechanism provided by DLC1/2 on BH3-only molecules may have a role in malignant transformation and drug resistance. This is the first demonstration that targeting this anti-apoptotic mechanism by an exogenous molecule shifts the balance towards apoptosis. Therefore, MVaf may be applied as an apoptosis-inducing tool for chemoresistant cancer cells, opening new avenues for future investigations in this field. These findings also raise the potential of developing novel therapeutic drugs based on the rational shown here, aiming at clinical application.

\section{Material and Methods}

Cell lines and culture. Murine melanoma cell lines B16-F10 (derived from C57BL/6J mouse, D/D, B/B), carrying WT MYO5A gene, and S91-6 (isolated from a DBA/2J mouse $d v / d v, b / b$ ), carrying the dilute MYO5A gene mutation were generously provided by Dr. J. Pawelek (Yale University, New Haven, CT, USA). Both cell lines were grown in Ham-F10 medium (Gibco-BRL, Gaithersburg, MD, USA) supplemented with $10 \%$ heat-inactivated fetal bovine serum (FBS; Invitrogen, Carlsbad, CA, USA) and antibiotics. Human melanoma cell lines WM35 and WM902 (derived from primary tumors in the radial and vertical growth phase, respectively) were generously provided by Dr. Meenhard Herlyn (The Wistar Institute Cancer Center). They were cultured in TU 2\% medium containing 80\% MCDB-153 (Sigma-Aldrich, St. Louis, MO, USA), 20\% Leibovitz's
L15 (Invitrogen), 2\% heat-inactivated FBS, $5 \mathrm{mg} / \mathrm{ml}$ insulin and $1.68 \mathrm{mM}$ $\mathrm{CaCl}_{2}$ (both from Sigma-Aldrich). ${ }^{48} \mathrm{MEFs}$ with double-knockout for $\mathrm{Bmf} / \mathrm{Bim}$

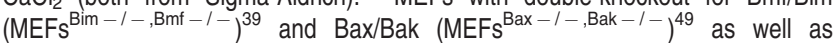
respective WT were kindly provided by Dr. Roger J Davis (Howard Hughes Medical Institute, Massachusetts Medical School). Also, MEFs with knockout for Bmf or Bim were kindly provided by Dr. Andreas Villunger (Innsbruck Medical University, Austria) and Professor Andreas Strasser (Walter and Eliza Hall Institute, Melbourne, Australia). All MEFs were cultured in Dulbecco's modified Eagle's medium; Invitrogen) supplemented with $10 \%$ heat-inactivated FBS, $1 \%$ non-essential amino acids (Invitrogen), 1\% L-glutamine, $50 \mu \mathrm{M} \beta$-mercaptoethanol, 20 mM HEPES and antibiotics. The human embryonic kidney cell line HEK293T/17 (CRL-11268 from ATCC) was cultured in Dulbecco's modified Eagle's medium low glucose (Invitrogen) supplemented with 10\% heat-inactivated FBS and antibiotics. All cell cultures were maintained at $37^{\circ} \mathrm{C}$ in a humidified atmosphere of $5 \% \mathrm{CO}_{2}-95 \%$ air.

\section{Expression constructs for MVaf and MVcf, and transfection.} Multiple alignments of the complete amino acid sequence from the three human class $\mathrm{V}$ myosin members were performed, by means of the ClustalW program, version 1.81 (Supplementary Figure S1-A). The lowest conserved regions among the three class $\mathrm{V}$ paralogs were selected, reasoning that their expression in mammalian cells would interfere with specific functions of each particular paralog only. Fragments of about 50 amino acids long were defined in order to exclude stretches of conserved sequences common to the three paralogs, and to have a size enough to bare the core of a protein-protein interaction motif. MVafs and MVcfs cloned to generate the fusion constructs are highlighted in Supplementary Figure S1-A, and are also represented by line diagrams positioned beneath the linear schematic for myosin-Va in Figure 1a. Myosin-Vb fragment was not included in this study. Three MVafs were generated. The first fragment, termed MVaf1, comprised amino acids 1276-1328 (53 amino acid residues) including parts of exons $A$ and $D$ and all of exon $B$, and $C$. Note that the selected region for MVaf1 corresponds to the core of the DLC2-binding region. ${ }^{15,31}$ Two additional constructs, termed MVaf2 and MVaf3 were generated in order to gain insights into the requirement of tissue-specific exons for any observed effect. Constructs MVaf2 and MVaf3 comprise amino acids 1255-1320 in NM_000259. MVaf2 is composed of 66 residues, including part of exon $A$, and complete exon $B$, and $C$, whereas MVaf3 encompasses 63 residues, excluding exon B. Such strategy aimed to survey possible connections between the cell death mechanism triggered by MVaf1 and DLC association. MVcf, termed MVcf, comprises amino acid residues 1179-1232 of the predicted human myosin-Vc sequence (NM_018728). All constructs had the correct sequences and expected fusion tags. EGFP-MVaf1 and EGFP (control) were immunoprecipitated from HEK-293 cell lysates with antiEGFP (Clontech, Mountain View, CA, USA). The expected molecular mass and integrity of the fusion proteins were confirmed by SDS-PAGE (Supplementary Figure S1-B) and western blot (Supplementary Figure S1-C) using anti-EGFP and anti-myosin-Va antibodies. An antibody generated to GST-MVaf1 recognizes endogenous brain myosin-Va (Supplementary Figure S1-D). The remaining recombinant proteins used in this work were also checked for integrity by SDSPAGE and western blotting (data not shown).

PCR products for MVaf, MVcf and other constructs were prepared using CDNA templates from different sources, such as human brain (MVaf1), HeLa (MVcf), 1205Lu melanoma cells (DLC2), B cells (Bmf), sk-mel melanoma cells (MVaf2 and MVaf3), and clone EST63321 from ATCC (DLC1). RT-PCR products were cloned into pUC18, pGEX2T, pGEX-4T-3 or plH 902 vectors (Amersham Biosciences, GE Healthcare, Uppsala, Sweden), as well as pEGFP-C1 and pDsRed1-C1 (Clontech). Transfections of B16-F10, S91 and WM cells were performed with the SuperFect transfection reagent (Qiagen, Valencia, CA, USA) according to the manufacturer's instructions. The transfection of HEK-293T cells was carried out using the calcium phosphate co-precipitation technique.

Cell proliferation and viability assays. B16-F10, S91-6 and WM melanoma cells were seeded on $35-\mathrm{mm}$ glass-bottom dishes $\left(7 \times 10^{4} \mathrm{cells} / \mathrm{dish}\right)$ for $24 \mathrm{~h}$, and transiently transfected as described above. Cultures $(n=5)$ were imaged (20 random fields of 1.6- $\mathrm{mm}^{2}$ each/dish) on a Fluorescence Stereomicroscope LEICA MZFL3 (Leica Microsystems GmbH, Wetzlar, Germany), after PI staining (Sigma-Aldrich, catalog no. P4170), using two channels of fluorescence (green and red). Cell count was performed after periods of 8, 18, 48 and $72 \mathrm{~h}$ (or $96 \mathrm{~h}$ ) post transfection. Viability was determined as the percentage of PI-positive dead cells, calculated relative to the total number of green fluorescent-transfected 
cells using the ImageJ software (http://rsb.info.nih.gov). Alternatively, cells were seeded on 24-well plates $\left(1.2 \times 10^{4}\right.$ cells/well), and cell counting was performed directly under microscopic examination ( $\times 40$ objective, Nikon Eclipse TS100), by scanning along five fixed lines.

Fluorescence and time-lapse microscopy; DNA fragmentation and active caspase-3 detection assays; cytochrome-c and Smac release assays. B16-F10 cells were cultured for $24 \mathrm{~h}$ on glass coverslips coated with poly-D-lysine (Sigma-Aldrich), transfected with pEGFPMVaf1 or pEGFP (control) and incubated for more 24 or $48 \mathrm{~h}$, when they were fixed, permeabilized and labeled with $2 \mu \mathrm{g} / \mathrm{ml}$ DAPI (Molecular Probes, Invitrogen, catalog no. D1306) and $0.002 \mathrm{U} / \mu \mathrm{l}$ Rhodamine-conjugated Phalloidin (Molecular Probes, Invitrogen, catalog no. R415), according to the manufacturer's protocol. For TUNEL assay, $48 \mathrm{~h}$ post transfection culture, dishes were gently shaken to bring to suspension loosely adhered cells, which were then harvested by centrifugation, resuspended in culture medium, distributed onto poly-D-lysinecoated coverslips and centrifuged again at $38 \times \mathrm{g}$ for $5 \mathrm{~min}$ at $4^{\circ} \mathrm{C}$ to accelerate cell adhesion. The TUNEL cell death assay was carried out using the 'In Situ Cell Death Detection Kit' TMR red (Roche Applied Science, Indianapolis, IN, USA, catalog no. 12156792910) according to manufacturer's instructions. Cells were imaged using a Zeiss Axiovert 100 inverted fluorescence microscope (Carl Zeiss, Jena, Germany), in 10 random fields per coverslip using two channels of fluorescence (green and red). The percentage of TUNEL-positive cells was calculated as the ratio between TUNEL-positive and total number of cells counted ( $\sim 150$ cells/coverslip). In order to monitor cytochrome-c release, B16-F10 cells grown on poly-D-lysine-coated coverslips, and expressing either EGFP-MVaf1 or EGFP were fixed and stained by immunocytochemistry with monoclonal anti-cytochrome-c primary antibody (BD Biosciences, San Jose, CA, USA) and Alexa Fluor 594 donkey anti-mouse IgG secondary antibody (Molecular Probes, Invitrogen Corp., Eugene, OR, USA). Coverslips were mounted in Fluoromount G (Electron Microscopy Sciences, Washington, PA, USA), imaged on an inverted fluorescence microscope as previously described, and analyzed using the Metamorph 4.1 program. The percentage of cells displaying a diffuse cytosolic cytochrome-c staining was calculated relative to the total number of green fluorescent cells. For time-lapse analysis of caspase-3 activity, B16-F10 cells grown on glass-bottom microwell dishes $(\Phi 35 \mathrm{~mm})$. coated with poly-D-lysine were treated with caspase-3 Fluorogenic Substrate IX (Calbiochem, San Diego, CA, USA) and (Z-DEVD)2-Rh110 for $10 \mathrm{~min}, 24 \mathrm{~h}$ post transfection. Cells were imaged in $\mathrm{CO}_{2}$-independent medium (Invitrogen), by confocal microscopy, using a Leica TCS-SP5 AOBS system, equipped with an incubator chamber. Red and green fluorescence images were captured in two channels: EGFP ex/em max $488 / 509 \mathrm{~nm}$; Rhodamine $110 \mathrm{ex} / \mathrm{em} \max 494 / 521 \mathrm{~nm}$. The distribution of the proapoptotic mitochondrial protein Smac was assessed in $24 \mathrm{~h}$ cultures of B16-F10, $\mathrm{MEF}^{\mathrm{WT}}$ and Bax/Bak double-knockout MEF cells, grown on coated glass-bottom microwell dishes $(\Phi 35 \mathrm{~mm})$ and co-transfected with pEGFP-MVaf1 (green) and pSmac-Cherry (red). Cells were time-lapse imaged in $\mathrm{CO}_{2}$-independent medium (Invitrogen), as described above, and those exhibiting morphological features of cell death were scored and represented as percentage of dead cells relative to the total number of green fluorescent cells. Ten independent time-lapses were assessed for each cell line. For the analysis of MVaf1 and DLC2 colocalization, live cells co-expressing DsRed-MVaf1 and EGFP-DLC2 were imaged at 24, 48, 72 and $96 \mathrm{~h}$ post transfection by confocal microscopy, as previously described.

Scanning electron microscopy. ForSEM, B16-F10 cells were cultured on Cellocate coverslips (Eppendorf, Hamburg, Germany) with grid square size of $55 \mu \mathrm{m}$. At $24 \mathrm{~h}$ post transfection, fluorescent cells on the Cellocate grid were imaged under fluorescence and bright-field microscopy, so that the grid coordinates were registered. They were then immediately fixed with $2 \%$ glutaraldehyde in PBS containing $1 \mathrm{mM} \mathrm{CaCl}_{2}$ and $0.5 \mathrm{mM} \mathrm{MgCl}_{2} \bullet 6 \mathrm{H}_{2} \mathrm{O}$, for $2 \mathrm{~h}$ at room temperature, rinsed in $0.1 \mathrm{M}$ sodium cacodylate buffer, $\mathrm{pH} 7.4$ and postfixed in $1 \%$ osmium tetroxide in the same buffer, for $2 \mathrm{~h}$ at room temperature, rinsed five times in deionized $\mathrm{H}_{2} \mathrm{O}$. Subsequently, samples were incubated with a saturated solution of thiocarbohydrazide for $10 \mathrm{~min}$ in the dark, rinsed five times in deionized $\mathrm{H}_{2} \mathrm{O}$, followed by $1 \%$ osmium tetroxide for $30 \mathrm{~min}$ in the dark and rinsed again. These steps were repeated once. Cells were dehydrated in a graded series of ethanol and critically point-dried with liquid $\mathrm{CO}_{2}$ in a BAL-TEC-CPD 030 CriticalPoint Dryer (Bal-Tec AG, Balzers, Liechtenstein), mounted on aluminum stubs with silver paint (EM Sciences) and coated with gold in a BAL-TEC SCD 050 Sputter Coater (BAL-TEC). Samples were examined in a JEOL JSM-5200 SEM.
Caspase-9 detection. Cell lysates from B16-F10 cells expressing either EGFP-MVaf1 or EGFP were collected at 24, 36, 48 and $60 \mathrm{~h}$ post transfection, combined, electrophoresed on $15 \%$ SDS-PAGE gel and measured by western blotting for detection of pro-caspase-9 and caspase-9, using pAb anti-caspase-9 (BD Biosciences, catalog no. 552036) and goat anti-rabbit IgG, HRP-conjugate (Pierce, Rockford, IL, USA) as secondary antibody. Quantification of the specific bands from the western blotting was measured by the ratio of pixel intensity using ImageJ gel analysis software (U. S. National Institutes of Health, Bethesda, MD, USA).

Pull-down assays. Bacterially expressed and purified GST-MVaf1, GSTMVcf, GST-MT (positive control), and GST (negative control) were used as bait in pull-down assays, whereas MBP-DLC2 or MBP (control) were used as prey. Bait proteins $(200 \mu \mathrm{g})$ were adsorbed to $50 \mu \mathrm{l}$ Glutathione-Sepharose 4B (Amersham Biosciences) for $1 \mathrm{~h}$, at $4^{\circ} \mathrm{C}$, on a rocker. Beads were washed thrice with cold PBS and prey proteins (200 or $800 \mu \mathrm{g}$ ) were incubated with bait-treated resin for $18 \mathrm{~h}$ at $4^{\circ} \mathrm{C}$ on a rocker. Beads were washed again and bound material was eluted from beads with $50 \mu \mathrm{l} \mathrm{SDS}$-PAGE sample buffer at $50^{\circ} \mathrm{C}$, using microSpin columns (Promega, San Luis Obispo, CA, USA) at $5000 \times g$ for $5 \mathrm{~min}$. Eluates were subjected to SDS-PAGE and western blotting, and were immunodetected by anti-myosin-Va MT (anti-MT), anti-GFP (Clontech), anti-Bcl2 (BD Biosciences, Franklin Lakes, NJ, USA), anti-DLC/PIN (Santa Cruz Biotechnology, Santa Cruz, CA, USA) or anti-DLC/LC-8, generated against Clamydomonas LC-8 R-4058. Pulldown assays were also performed with cell lysates from HEK-293T expressing different EGFP fusion proteins or EGFP alone (negative control).

RNA extraction and Northern blot analysis. Total RNA was extracted from cell cultures of human melanoma cell lines WM35 and WM902 by using the TRI Reagent (Sigma-Aldrich) according to the manufacturer's instructions, and Northern blot analysis was performed according to Nature Methods 2: 997-998 (2005), in order to assess MYO5A and DLC2 gene expression profiles at the transcript level, while $\beta$-actin mRNA was used as the loading control. Quantification of the specific bands from the northern blotting was measured by the ratio of pixel intensity (relative signal) using ImageJ gel analysis software.

\section{Melanoma model obtained by inoculation of B16 murine melanoma cell line.}

Animals. C57BL/6J mice of eight weeks were kept on a $12 \mathrm{~h} / 12 \mathrm{~h}$ light/dark cycle, at a normal $\left(24^{\circ} \mathrm{C}\right)$ animal house temperature, humidity above $55 \%$, were fed ad libitum and had free access to water. The experimental protocol was approved by the Faculty of Medicine of Ribeirão Preto Bioethical Committee (process number 25000.079855/2006-96).

Selection of transduced melanoma cells and transplantation in mice. Cultures of retrovirus packaging cells (Retropack PT67, Clontech) or HEK293T cells were transfected with plasmids pRevTetOn/pRevTRE (bearing sequences of EGFPMVaf1) and pLVXTetON/pLVXTightpuro bearing EGFP, respectively. TetON vector systems were from Clontech Laboratories, Inc., Mountain View, CA, USA. Viruses were generated, amplified and titrated according to the manufacturer's manual. B16-F10 murine melanoma cells were grown to $50-70 \%$ confluence and infected with recombinant virus. EGFP- or EGFP-MVaf1-expressing cells were sorted after brief induction with doxycycline $(1 \mu \mathrm{g} / \mathrm{ml}$ for $24 \mathrm{~h}$, half of the regular concentration and time required for peak expression). Sorting was done using a cell sorter flow cytometer (FACsAria from BD Biosciences, San Jose, CA). A mixed population of green fluorescent cells was sorted and cultured for expansion of the cells in doxycycline-free complete medium. These cells, representing not a clone, but a mixed population of transduced cells (referred to as 'Sorted' EGFP-MVaf1 cells) were used for transplantation in vivo for tumorigenicity assays (Figures $6 \mathrm{a}, \mathrm{b}$ and d). Also we generated two independent clonal lineages, $\mathrm{C} 3$ and $\mathrm{G} 3$, which were used for tumorigenicity assays to determine survival rates (Figure 6c). C57BL/6J mice were shaved dorsally and received subcutaneous injections of B16-F10 melanoma cells $\left(5 \times 10^{5}\right.$ cells in PBS), on each posterior flank. The right flanks were injected with B16-F10 melanoma cells transduced with EGFP-MVaf1, whereas the left flanks were injected with the same cell line transduced with EGFP (control). The animals were divided into two groups ( $n=$ 8/group), one of which received doxycycline in the drinking water $(200 \mu \mathrm{g} / \mathrm{ml}$ in $1 \%$ sucrose), immediately after injections and along all the study. Volumetric measurement of tumors was performed externally by using a calliper. Animals were monitored for a 30-day period or longer, until euthanasia. Experimental endpoints were preceded by limiting clinical signs such as consistent weight loss, 
apathy, lethargy, and impaired respiration. A limitation noted was relatively low expression levels provided by the TetON system in comparison to plasmidial transfections with the Qiagen SuperFect reagent.

Clonogenic assay of cells in vitro. Approximately 600 cells (WT B16-F10 cells or stably transduced B16-F10 cells) were seeded in 60-mm dishes and treated with $2 \mu \mathrm{g} / \mathrm{ml}$ doxycycline. Colonies were fixed with paraformaldehyde $(4.0 \% \mathrm{v} / \mathrm{v}$ in PBS) for $20 \mathrm{~min}$, stained with crystal violet $(0.2 \% \mathrm{w} / \mathrm{v}$ in PBS) and washed with $\mathrm{H}_{2} \mathrm{Odd}$. Plates were photographed and colonies were counted using the software Image J v.1.42q.

Statistical analysis. Results are presented as the mean \pm S.E.M. Statistically significant difference between two means was assessed by the Student's $t$-test. Significant differences of growth parameters between two or more groups were assessed by two-way ANOVA followed by Bonferroni post hoc test. The level of significance was set at $1 \%(P<0.01)$. Statistical analysis of tumor growth experiments was performed using the unpaired two-tailed Student's $t$-test to assess the significance of the mean difference. Differences between designated groups compared with the control group were considered significant at values of $P<0.05$. All analyses were performed with GraphPad Prism 5.0 (GraphPad Software, San Diego, CA, USA).

\section{Conflict of Interest}

The authors declares no conflict of interest.

Acknowledgements. We are grateful to technicians Silmara Reis Banzi and Domingos E Pitta for expert technical assistance with protein purification and antibody production; to Maria Dolores S Pereira, Maria Teresa P Maglia and José Augusto Maulin for assistance with electron microscopy; to Dr. Marcia Graeff for the technical supervision on the confocal microscopy analyses in the 'Centro Multiusuário de Microscopia Confocal-FMRP-USP'; to Benedita $O$ de Souza for general lab assistance; and to Domingos de Souza for biotery assistance. We also thank Patricia Viana Bonini Palma and Camila Cristina de Oliveira Menezes Bonaldo for help with cell sorting at the Flow Cytometry Lab, Hemotherapy Center of Ribeirão Preto. We are thankful to Dr. Stanley J Korsmeyer (Howard Hughes Medical Institute, Departments of Pathology and Medicine, Harvard Medical School, Dana-Farber Cancer Institute, Boston, Massachusetts 02115,USA) for the Bcl2 construct in the pGEX-4T-3 vector; Dr. Foued S Espindola (University Federal of Uberlândia, Uberlândia MG, Brazil) for the cDNA clone EST63321 obtained from ATCC and anti-DLC Clamydomonas R-4058; Dr. David N Levy (New York University, New York, NY, USA) for helping us with the construction of vector pLVXEGFP. We are also grateful to the following researchers for providing cell lines: Dr. John Pawelek (Yale University, New Haven, CT, USA), Dr. Meenhard Herlyn (The Wistar Institute Cancer Center), Dr. Roger J Davis (Howard Hughes Medical Institute, Massachusetts Medical School), Dr. Andreas Villunger (Innsbruck Medical University, Austria) and Professor Andreas Strasser (Walter and Eliza Hall Institute, Melbourne, Australia). This work was supported by grants from Fundação de Amparo à Pesquisa do Estado de São Paulo (FAPESP \#2009/50167-3 - PI: EME), CNPq (\# 401322/2005-0 - PI: EME) and FAEPA. CAPES provided a doctorate fellowship to TCIT and CPA. FAPESP provided a technical fellowship to APBP, master fellowship to DDA and DMT, doctorate fellowship to JFS and EVP, postdoctoral fellowship to JFS and to DPSLM. CNPq provided a research fellowship to $E M E$, master and doctorate to $A C B$, international doctorate fellowship to $A C B$ and to CPA, undergraduate student fellowship to FONJ, and post-doctorate fellowship to JFS. We thank Ricardo GP Ramos and Roy E Larson for critical reading of the revised version.

1. Strasser A, Puthalakath H, Bouillet $P$, Huang DC, O'Connor L, O'Reilly LA et al. The role of bim, a proapoptotic BH3-only member of the Bcl-2 family in cell-death control. Ann N Y Acad Sci 2000; 917: 541-548.

2. Happo L, Strasser A, Cory S. BH3-only proteins in apoptosis at a glance. J Cell Sci 2012 125: $1081-1087$.

3. Rapali P, Szenes Á, Radnai L, Bakos A, Pál G, Nyitray L. DYNLL/LC8: a light chain subunit of the dynein motor complex and beyond. FEBS J 2011; 278: 2980-2996.

4. Espindola FS, Suter DM, Partata LB, Cao T, Wolenski JS, Cheney RE et al. The light chain composition of chicken brain myosin-Va: calmodulin, myosin-II essential light chains, and 8-kDa dynein light chain/PIN. Cell Motil Cytoskeleton 2000; 47: 269-281.
5. Naisbitt S, Valtschanoff J, Allison DW, Sala C, Kim E, Craig AM et al. Interaction of the postsynaptic density-95/guanylate kinase domain-associated protein complex with a light chain of myosin-V and dynein. J Neurosci 2000; 20: 4524-4534.

6. Rodriguez OC, Cheney RE. Human myosin-Vc is a novel class $V$ myosin expressed in epithelial cells. J Cell Sci 2002; 115: 991-1004.

7. Larson RE. Myosin-V: a class of unconventional molecular motors. Braz J Med Biol Res 1996; 29: 309-318

8. Reck-Peterson SL, Provance DW Jr, Mooseker MS, Mercer JA. Class V myosins. Biochim Biophys Acta 2000; 1496: 36-51.

9. Hammer JA 3rd, Sellers JR. Walking to work: roles for class V myosins as cargo transporters. Nat Rev Mol Cell Biol 2011; 13: 13-26.

10. Puthalakath H, Villunger A, O'Reilly LA, Beaumont JG, Coultas L, Cheney RE et al. Bmf: a proapoptotic $\mathrm{BH}$-only protein regulated by interaction with the myosin $\mathrm{V}$ actin motor complex, activated by anoikis. Science 2001; 293: 1829-1832.

11. Puthalakath H, Huang DC, O'Reilly LA, King SM, Strasser A. The proapoptotic activity of the $\mathrm{Bcl}-2$ family member $\mathrm{Bim}$ is regulated by interaction with the dynein motor complex. Mol Cell 1999; 3: 287-296

12. Hunt A, Evan G. Apoptosis. Till death us do part. Science 2001; 293: 1784-1785

13. Simpson CD, Anyiwe K, Schimmer AD. Anoikis resistance and tumour metastasis. Cancer Lett 2008; 272: 177-185.

14. Taddei ML, Giannoni E, Fiaschi T, Chiarugi P. Anoikis: an emerging hallmark in health and diseases. J Pathol 2012; 226: 380-393.

15. Hódi Z, Németh AL, Radnai L, Hetényi C, Schlett K, Bodor A et al. Alternatively spliced exon $B$ of myosin $\mathrm{Va}$ is essential for binding the tail-associated light chain shared by dynein. Biochemistry 2006; 45: 12582-12595.

16. Day CL, Puthalakath H, Skea G, Strasser A, Barsukov I, Lian LY et al. Localization of dynein light chains 1 and 2 and their pro-apoptotic ligands. Biochem J2004; 377: 597-605.

17. Zhu Z, Sanchez-Sweatman O, Huang X, Wiltrout R, Khokha R, Zhao Q et al. Anoikis and metastatic potential of cloudman S91 melanoma cells. Cancer Res 2001; 61: 1707-1716.

18. Van Cruchten S, Van Den Broeck W. Morphological and biochemical aspects of apoptosis, oncosis and necrosis. Anat Histol Embryol 2002; 31: 214-223.

19. VanBrocklin MW, Verhaegen M, Soengas MS, Holmen SL. Mitogen-activated protein kinase inhibition induces translocation of Bmf to promote apoptosis in melanoma. Cancer Res 2009; 69: 1985-1994.

20. Shao $Y$, Aplin AE. BH3-only protein silencing contributes to acquired resistance to PLX4720 in human melanoma. Cell Death Differ 2012; 19: 2029-2039.

21. Du YC, Lewis BC, Hanahan D, Varmus H. Assessing tumour progression factors by somatic gene transfer into a mouse model: Bcl-xL promotes islet tumour cell invasion. PLOS Biol 2007; 5: e276.

22. Galland F, Lacroix L, Saulnier P, Dessen P, Meduri G, Bernier M et al. Differential gene expression profiles of invasive and non-invasive non-functioning pituitary adenomas based on microarray analysis. Endocr Relat Cancer 2010; 17: 361-371.

23. Lan L, Han H, Zuo H, Chen Z, Du Y, Zhao W et al. Upregulation of myosin Va by Snail is involved in cancer cell migration and metastasis. Int J Cancer 2010; 126: 53-64.

24. van Diepen MT, Parsons M, Downes CP, Leslie NR, Hindges R, Eickholt BJ. MyosinV controls PTEN function and neuronal cell size. Nat Cell Biol 2009; 11: 1191-1196.

25. Rudolf $\mathrm{R}$, Bittins $\mathrm{CM}$, Gerdes $\mathrm{HH}$. The role of myosin $\mathrm{V}$ in exocytosis and synaptic plasticity. J Neurochem 2011; 116: 177-191.

26. Chen $Y$, Wang Y, Zhang J, Deng Y, Jiang L, Song E et al. Rab10 and myosin-Va mediate insulin-stimulated GLUT4 storage vesicle translocation in adipocytes. J Cell Biol 2012; 198 : 545-560.

27. Adekola K, Rosen ST, Shanmugam M. Glucose transporters in cancer metabolism. Curr Opin Oncol 2012; 24: 650-654.

28. Espreafico EM, Coling DE, Tsakraklides V, Krogh K, Wolenski JS, Kalinec G et al. Localization of myosin-V in the centrosome. Proc Natl Acad Sci USA 1998: 95: 8636-8641.

29. Pranchevicius MC, Baqui MM, Ishikawa-Ankerhold HC, Lourenço EV, Leão RM, Banzi SR et al. Myosin Va phosphorylated on Ser1650 is found in nuclear speckles and redistributes to nucleoli upon inhibition of transcription. Cell Motil Cytoskeleton 2008; 65: 441-456.

30. Salerno VP, Calliari A, Provance DW Jr, Sotelo-Silveira JR, Sotelo JR, Mercer JA. MyosinVa mediates RNA distribution in primary fibroblasts from multiple organs. Cell Motil Cytoskeleton 2008; 65: 422-433.

31. Letai A, Bassik MC, Walensky LD, Sorcinelli MD, Weiler S, Korsmeyer SJ et al. Distinct $\mathrm{BH} 3$ domains either sensitize or activate mitochondrial apoptosis, serving as prototype cancer therapeutics. Cancer Cell 2002; 2: 183-192.

32. Gavathiotis E, Suzuki M, Davis ML, Pitter K, Bird GH, Katz SG et al. BAX activation is initiated at a novel interaction site. Nature 2008; 455: 1076-1081.

33. Wagner W, Fodor E, Ginsburg A, Hammer JA 3rd. The binding of DYNLL2 to myosin Va requires alternatively spliced exon $B$ and stabilizes a portion of the myosin's coiled-coil domain. Biochemistry 2006; 45: 11564-11577.

34. Vadlamudi RK, Bagheri-Yarmand R, Yang Z, Balasenthil S, Nguyen D, Sahin AA et al. Dynein light chain 1, a p21-activated kinase 1-interacting substrate, promotes cancerous phenotypes. Cancer Cell 2004; 5: 575-585.

35. Lu J, Sun Q, Chen X, Wang H, Hu Y, Gu J. Identification of dynein light chain 2 as an interaction partner of p21-activated kinase 1. Biochem Biophys Res Commun 2005; 331 : 153-158.

36. Lei K, Davis RJ. JNK phosphorylation of Bim-related members of the Bcl2 family induces Bax-dependent apoptosis. Proc Natl Acad Sci USA 2003; 100: 2432-2437. 
37. Schmelzle T, Mailleux AA, Overholtzer M, Carroll JS, Solimini NL, Lightcap ES et al. Functional role and oncogene-regulated expression of the BH3-only factor Bmf in mammary epithelial anoikis and morphogenesis. Proc. Natl. Acad. Sci. USA 2007; 104: 3787-3792.

38. Kepp O, Gottschalk K, Churin Y, Rajalingam K, Brinkmann V, Machuy N et al. Bim and Bmf synergize to induce apoptosis in Neisseria gonorrhoeae infection. PLoS Pathog 2009; 5 : e1000348.

39. Hübner A, Cavanagh-Kyros J, Rincon M, Flavell RA, Davis RJ. Functional Cooperation of the proapoptotic Bcl2 family proteins Bmf and Bim in vivo. Mol Cell Biol 2010; 30: 98-105.

40. van de Water B, Nagelkerke JF, Stevens JL. Dephosphorylation of focal adhesion kinase (FAK) and loss of focal contacts precede caspase-mediated cleavage of FAK during apoptosis in renal epithelial cells. J Biol Chem 1999; 274: 13328-13337.

41. Ferro ES, Hyslop S, Camargo AC. Intracellullar peptides as putative natural regulators of protein interactions. J Neurochem 2004; 94: 769-777.

42. Larribere L, Hilmi C, Khaled M, Gaggioli C, Bille K, Auberger P et al. The cleavage of microphthalmia-associated transcription factor, MITF, by caspases plays an essential role in melanocyte and melanoma cell apoptosis. Genes Dev 2005; 19: 1980-1985.

43. Mazumder S, Plesca D, Almasan A. A jekyll and hyde role of cyclin E in the genotoxic stress response: switching from cell cycle control to apoptosis regulation. Cell Cycle 2007; 6: $1437-1442$.

44. Espreafico EM, Cheney RE, Matteoli M, Nascimento AA, De Camilli PV, Larson RE et al. Primary structure and cellular localization of chicken brain myosin-V (p190), an unconventional myosin with calmodulin light chains. J Cell Biol 1992; 119: 1541-1557.
45. Coelho MV, Larson RE. $\mathrm{Ca}(2+)$-dependent phosphorylation of the tail domain of myosin-V, a calmodulin-binding myosin in vertebrate brain. Braz J Med Biol Res 1993; 26: 465-472.

46. Nascimento AA, Cheney RE, Tauhata SB, Larson RE, Mooseker MS. Enzymatic characterization and functional domain mapping of brain myosin-V. J Biol Chem 1996; 271: 17561-17569.

47. Casaletti L, Tauhata SB, Moreira JE, Larson RE. Myosin-Va proteolysis by $\mathrm{Ca}^{2+} /$ calpain in depolarized nerve endings from rat brain. Biochem Biophys Res Commun 2003; 308: 159-164.

48. Balaban G, Herlyn M, Guerry D IV, Bartolo R, Koprowski H, Clark WH et al. Cytogenetics of human malignant melanoma and premalignant lesions. Cancer Genet Cytogenet 1984; 11 : 429-439.

49. Lindsten T, Ross AJ, King A, Zong WX, Rathmell JC, Shiels HA et al. The combined functions of proapoptotic Bcl-2 family members Bak and Bax are essential for normal development of multiple tissues. Mol. Cell 2000; 6: 1389-1399.

(i) (2) $\Theta$ Cell Death and Disease is an open-access journal published by Nature Publishing Group. This work is licensed under the Creative Commons Attribution-NonCommercial-No Derivative Works 3.0 Unported License. To view a copy of this license, visit http://creativecommons.org/licenses/by-nc-nd/3.0/

Supplementary Information accompanies this paper on Cell Death and Disease website (http://www.nature.com/cddis) 